\title{
The classification of toroidal Dehn surgeries on Montesinos knots
}

\author{
YING-QING WU
}

\begin{abstract}
Exceptional Dehn surgeries have been classified for two-bridge knots and Montesinos knots of length at least 4 . In this paper, we classify all toroidal Dehn surgeries on Montesinos knots of length 3 .
\end{abstract}

\section{Introduction}

A Dehn surgery on a hyperbolic knot $K$ along a non-trivial slope $\delta$ is said to be exceptional if the resulting manifold $K_{\delta}$ is either reducible, toroidal or a small Seifert fibered manifold. By the Geometrization Conjecture proved by Perelman [1], a non-trivial surgery is exceptional if and only if $K_{\delta}$ is non-hyperbolic. By Thurston's Hyperbolic Surgery Theorem, all but finitely many Dehn surgeries on a hyperbolic knot produce hyperbolic manifolds, hence there are only finitely many exceptional surgeries.

It is known that there are no exceptional surgeries on Montesinos knots of length at least four [5]. Exceptional surgeries for two-bridge knots have been classified in [2]. Thus length 3 knots are the only ones among the Montesinos knots, which have not been settled. In this paper, we will classify toroidal surgeries for such knots. See Theorems 1.1 and 1.2 below. By [3] there is no reducible surgery on a hyperbolic Montesinos knot because it is strongly invertible. It remains a challenging open problem to determine all small Seifert fibred surgeries on Montesinos knots of length 3.

Hatcher and Oertel [4] have an algorithm to determine all boundary slopes of a given Montesinos knot. We will therefore focus on finding all length 3 knots such that some of their boundary slopes are toroidal slopes. Each incompressible surface in the exterior of $K$ corresponds to three "allowable edgepaths" $\gamma_{1}, \gamma_{2}, \gamma_{3}$. We will define an Euler number for allowable edge paths, and show that if $F\left(\gamma_{1}, \gamma_{2}, \gamma_{3}\right)$ is a punctured torus then one of the $\gamma_{i}$ must have non-negative Euler number. We then analyze the graph of Hatcher-Oertel (figure 1), and show that the ending point of one of the above $\gamma_{i}$ must lie in a subgraph consisting of seven edges. This breaks the 


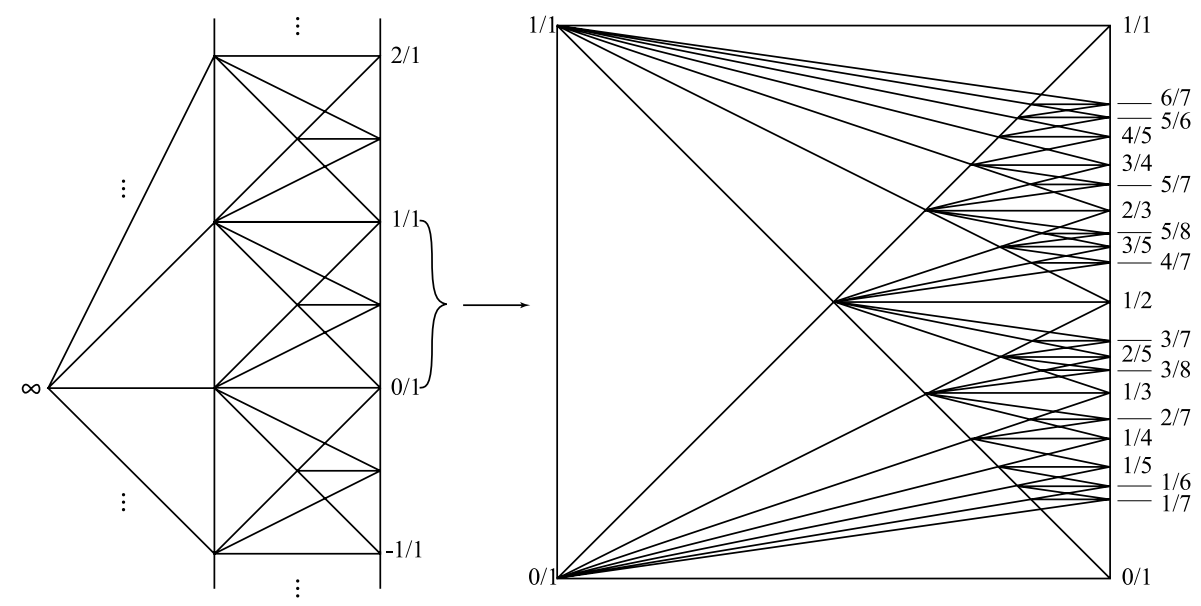

Figure 1:

problem down to several different cases. We will then use the properties of allowable edge paths to find all possible solutions in each case.

Two knots are considered equivalent if there is a (possibly orientation reversing) homeomorphism of $S^{3}$ sending one knot to the other. Thus $K_{1}$ is equivalent to $K_{2}$ if $K_{1}$ is isotopic to $K_{2}$ or its mirror image. Similarly, if $N\left(K_{i}\right)$ is a neighborhood of $K_{i}$ and $\delta_{i}$ is a slope on $\partial N\left(K_{i}\right)$, then $\left(K_{1}, \delta_{1}\right)$ is equivalent to $\left(K_{2}, \delta_{2}\right)$ if there is a homeomorphism of $S^{3}$ sending $N\left(K_{1}\right)$ to $N\left(K_{2}\right)$ and $\delta_{1}$ to $\delta_{2}$. The following is the classification theorem for toroidal boundary slopes of Montesinos knots of length 3 . Some knots are listed more than once, with different boundary slopes, which means that they admit more than one toroidal surgery. The variable $\bar{u}$ is the $u$ coordinate of the ending points of the edge paths, which will be defined in Section 2. Note that some knots may have the same toroidal boundary slope at different $\bar{u}$ values, in which case we will only list one $\bar{u}$ value.

Theorem 1.1. Let $K$ be a hyperbolic Montesinos knot of length 3 , let $E(K)=S^{3}-\operatorname{Int} N(K)$, and let $\delta$ be a slope on $\partial E(K)$. Then $E(K)$ contains an essential surface $F$ of genus one with boundary slope $\delta$ if and only if $(K, \delta)$ is equivalent to one of the pairs in the following list.

(1) $K=K\left(1 / q_{1}, 1 / q_{2}, 1 / q_{3}\right), q_{i}$ odd, $\left|q_{i}\right|>1, \delta=0 ; \bar{u}=1$.

(2) $K=K\left(1 / q_{1}, 1 / q_{2}, 1 / q_{3}\right), q_{1}$ even, $q_{2}, q_{3}$ odd, $\left|q_{i}\right|>1, \delta=2\left(q_{2}+q_{3}\right)$; $\bar{u}=1$. 
(3) $K=K(-1 / 2,1 / 3,1 /(6+1 / n)), n \neq 0,-1, \delta=16$ if $n$ is odd, and 0 if $n$ is even; $\bar{u}=6$.

(4) $K=K(-1 / 3,-1 /(3+1 / n), 2 / 3), n \neq 0,-1, \delta=-12$ when $n$ is odd, and $\delta=4$ when $n$ is even; $\bar{u}=3$.

(5) $K=K(-1 / 2,1 / 5,1 /(3+1 / n))$, n even, and $n \neq 0, \delta=5-2 n ; \bar{u}=3$.

(6) $K=K(-1 / 2,1 / 3,1 /(5+1 / n))$, $n$ even, and $n \neq 0, \delta=1-2 n ; \bar{u}=3$.

(7) $K=K(-1 /(2+1 / n), 1 / 3,1 / 3), n$ odd, $n \neq-1, \delta=2 n ; \bar{u}=2$.

(8) $K=K(-1 / 2,1 / 3,1 /(3+1 / n)), n$ even, $n \neq 0, \delta=2-2 n ; \bar{u}=2$.

(9) $K=K(-1 / 2,2 / 5,1 / 9), \delta=15 ; \bar{u}=5$.

(10) $K=K(-1 / 2,2 / 5,1 / 7), \delta=12 ; \bar{u}=4$.

(11) $K=K(-1 / 2,1 / 3,1 / 7), \delta=37 / 2 ; \bar{u}=2.5$.

(12) $K=K(-2 / 3,1 / 3,1 / 4), \delta=13 ; \bar{u}=2.5$.

(13) $K=K(-1 / 3,1 / 3,1 / 7), \delta=1 ; \bar{u}=2.5$.

For each case in Theorem 1.1 , the candidate system $\left(\gamma_{1}, \gamma_{2}, \gamma_{3}\right)$ is given in the proofs of the lemmas, hence it is straightforward using the algorithm of Hatcher-Oertel to calculate the boundary slope of $F\left(\gamma_{1}, \gamma_{2}, \gamma_{3}\right)$ and show that it is an incompressible toroidal surface. For each individual knot this can also be verified using a computer program of Dunfield [16]. We will therefore concentrate on showing the "only if" part, that is, if the exterior of $K$ has an incompressible toroidal surface with boundary slope $\delta$ then $(K, \delta)$ must be one of those in the list.

In general, the existence of a toroidal incompressible surface $F$ with boundary slope $\delta$ in the exterior of a knot $K$ does not guarantee that $K_{\delta}$ is toroidal, because the corresponding closed surface $\hat{F}$ may be compressible in $K_{\delta}$. However, the following theorem shows that this does not happen for Montesinos knots of length 3; hence the above theorem actually gives a classification of all toroidal surgeries for Montesinos knots of length 3.

Theorem 1.2. Let $K$ be a hyperbolic Montesinos knot of length 3, and let $\delta$ be a slope on $T=\partial N(K)$. Then $K_{\delta}$ is toroidal if and only if $(K, \delta)$ is equivalent to one of those in the list of Theorem 1.1.

Together with [2] and [5], this gives a complete classification of toroidal surgeries on all Montesinos knots. The following are some of the consequences. 
(1) The only non-integral toroidal surgery on a Montesinos knot is the $37 / 2$ surgery on $K(-1 / 2,1 / 3,1 / 7)$.

(2) No Montesinos knot admits more than three toroidal surgeries, and the figure 8 knot and $K(-1 / 2,1 / 3,1 / 7)$ are the only ones admitting three toroidal surgeries.

(3) By [2], a 2-bridge knot admits exactly two toroidal surgeries if and only if it is associated to the rational number $1 /(2+1 / n)$ for some $|n|>2$. By checking the list in Theorem 1.1 for knots which are listed more than once, we see that $K\left(t_{1}, t_{2}, t_{3}\right)$ admits exactly two toroidal surgeries if and only if it is equivalent to one of the following 5 knots.

$$
\begin{array}{lc}
K(-1 / 2,1 / 3,2 / 11), & \delta=0 \text { and }-3 ; \\
K(-1 / 3,1 / 3,1 / 3), & \delta=0 \text { and } 2 ; \\
K(-1 / 3,1 / 3,1 / 7), & \delta=0 \text { and } 1 ; \\
K(-2 / 3,1 / 3,1 / 4), & \delta=12 \text { and } 13 ; \\
K(-1 / 3,-2 / 5,2 / 3), & \delta=4 \text { and } 6 .
\end{array}
$$

(4) A toroidal essential surface $F$ in Theorem 1.1 has at most four boundary components. In case (1) of Theorem $1.1 F$ is a Seifert surface with a single boundary component. In all other cases $F$ is a separating surface and the result follows from the proof of Theorem 1.2.

(5) Also follows from results of Gordon and Luecke [6] and EudaveMunoz [7], which classified non-integral toroidal surgeries on all knots in $S^{3}$. There are many other interesting results about toroidal Dehn surgery, see for example [8-15].

The paper is organized as follows. In Section 2 we give a brief introduction to some definitions and results of Hatcher and Oertel in [4], then define and explore the properties of Euler numbers $e(\gamma)$ for any edge path in the Hatcher-Oertel graph $\mathcal{D}$ shown in figure 1 . It will be shown that if $F\left(\gamma_{1}, \gamma_{2}, \gamma_{3}\right)$ is a punctured torus then up to equivalence the ending point $v_{1}$ of $\gamma_{1}$ must lie on the subgraph $G$ in figure 4 . Sections 3,4 and 5 discuss the cases that $v_{1}$ lies on a horizontal edge in $G$, and Section 6 deals with the remaining cases. The proofs of Theorems 1.1 and 1.2 will be given in Section 7.

\section{Preliminaries}

In this section we first give a brief introduction to some results of HatcherOertel in [4]. We will then define Euler numbers for points and edge paths on the Hatcher-Oertel diagram $\mathcal{D}$, and show how they are related to the Euler 
characteristic of the corresponding surfaces. The main result is Theorem 2.8, which will play a key role in finding Montesinos knots which admit toroidal surgeries.

\subsection{The diagram $\mathcal{D}$}

The diagram $\mathcal{D}$ of Hatcher-Oertel is a two-complex on the plane $\mathbb{R}^{2}$ consisting of vertices, edges and triangular faces described as follows. See figure 1, which is the same as [4, figure 1.3]. Unless otherwise stated, we will always write a rational number as $p / q$, where $p, q$ are coprime integers, and $q>0$.

(1) To each rational number $y=p / q$ is associated a vertex $\langle y\rangle$ in $\mathcal{D}$, which has Euclidean coordinates $(x, y)=((q-1) / q, p / q)$.

(2) For each rational number $y=p / q$, there is also an "ideal" vertex $\langle y\rangle_{0}$ with Cartesian coordinates $(1, p / q)$.

(3) There is a vertex $\infty=1 / 0$ located at $(-1,0)$.

(4) There is an edge $E=\left\langle p / q, p^{\prime} / q^{\prime}\right\rangle$ connecting $\langle p / q\rangle$ to $\left\langle p^{\prime} / q^{\prime}\right\rangle$ if and only if $\left|p q^{\prime}-p^{\prime} q\right|=1$. Thus for example, there is an edge connecting $\infty$ to each vertex $\langle p / 1\rangle$, and there is an edge connecting $\langle p / q\rangle$ to $\langle 0\rangle$ if and only if $p= \pm 1$.

(5) For each rational number $y$ there is a horizontal edge $L(y)$ connecting $\langle y\rangle$ to the ideal vertex $\langle y\rangle_{0}$.

(6) A face of $\mathcal{D}$ is a triangle bounded by three non-horizontal edges of $\mathcal{D}$.

Note that a non-horizontal line segment in the figure with one endpoint on an ideal vertex (i.e., a vertex on the vertical line $x=1$ ) is not an edge of $\mathcal{D}$. It is a union of infinitely many edges of $\mathcal{D}$ and contains infinitely many vertices of $\mathcal{D}$. Similarly a triangle $\Delta$ in the figure is not a face of $\mathcal{D}$ if it contains a horizontal edge because there are edges in the interior of $\Delta$. Actually in this case $\Delta$ is a union of infinitely many faces of $\mathcal{D}$. On the other hand, if all three vertices of a triangle $\Delta$ in the figure are non-ideal vertices of $\mathcal{D}$, and if all three boundary edges of $\Delta$ are edges of $\mathcal{D}$ as defined above, then $\Delta$ is a face of $\mathcal{D}$; in particular, its interior contains no other edges or vertices of $\mathcal{D}$.

\subsection{Allowable edge paths, candidate systems and candidate surfaces}

An edge path $\gamma$ in $\mathcal{D}$ is a piecewise linear path in the one-skeleton of $\mathcal{D}$. Note that the endpoints of $\gamma$ may not be vertices of $\mathcal{D}$. An edge path $\gamma$ is a constant path if its image is a single point. 
Let $K=K\left(t_{1}, t_{2}, t_{3}\right)$ be a Montesinos knot of length 3 . Let $\gamma_{1}, \gamma_{2}, \gamma_{3}$ be three edge paths in $\mathcal{D}$. According to $[4$, p. 457], we say that the three edge paths form a candidate system for $K\left(t_{1}, t_{2}, t_{3}\right)$ if they satisfy the following conditions:

(1) The starting point of $\gamma_{i}$ is on the horizontal edge $L\left(t_{i}\right)$, and if this starting point is not the vertex $\left\langle t_{i}\right\rangle$ then $\gamma_{i}$ is a constant path.

(2) $\gamma_{i}$ is minimal in the sense that it never stops and retraces itself, or goes along two sides of a triangle of $\mathcal{D}$ in succession.

(3) The ending points of $\gamma_{i}$ are rational points $\mathcal{D}$ which all lie on one vertical line and whose vertical coordinates add up to zero.

(4) $\gamma_{i}$ proceeds monotonically from right to left, "monotonically" in the weak sense that motion along vertical edges is permitted.

Each $\gamma_{i}$ above is called an allowable edge path. By definition, an allowable edge path must be of one of the following three types:

(1) A constant path on a horizontal edge, possibly at a vertex of $\mathcal{D}$.

(2) An edge path with both endpoints on vertices of $\mathcal{D}$.

(3) An edge path starting from a vertex of $\mathcal{D}$ and ending in the interior of a non-horizontal edge.

For each candidate system, one can construct a surface $F=F\left(\gamma_{1}, \gamma_{2}, \gamma_{3}\right)$ in the exterior of $K$, called a candidate surface. We refer the reader to [4, p. 457] for the construction of the surface. Denote by $\hat{F}=\hat{F}\left(\gamma_{1}, \gamma_{2}, \gamma_{3}\right)$ the corresponding closed surface obtained by capping off each boundary component of $F$ with a disk. Let $N(K)$ be a regular neighborhood of $K$. If $\delta$ is the boundary slope of $F$ on $\partial N(K)$ then $\hat{F}$ is a closed surface in the manifold $K_{\delta}$ obtained by $\delta$ surgery on $K$.

When $\gamma_{i}$ ends at $\langle\infty\rangle$ there may also be some "augmented" candidate surface, but fortunately this does not happen for Montesinos knots of length 3. The following is [4, Proposition 1.1].

Proposition 2.1. Every incompressible, $\partial$-incompressible surface in $S^{3}-$ $K$ having non-empty boundary of finite slope is isotopic to one of the candidate surfaces.

To find all toroidal surgeries on Montesinos knots of length 3 , it suffices to find all candidate systems $\left(\gamma_{1}, \gamma_{2}, \gamma_{3}\right)$ such that $\hat{F}=\hat{F}\left(\gamma_{1}, \gamma_{2}, \gamma_{3}\right)$ is a torus. By Theorem 1.2 all toroidal $\hat{F}$ are incompressible. 


\subsection{The $u$-coordinate of a point and the length of an edge path}

Any rational point $(x, y)$ in the diagram represents some curve system $(a, b, c)$ on a four-punctured sphere as shown in figure 2. (When $c$ is negative, reverse the tangency of the train track, and relabel $c$ by $-c$.) The parameters $(a, b, c)$ and $(x, y)$ are related as follows.

$$
y=\frac{c}{a+b}, \quad x=\frac{b}{a+b}
$$

See $[4$, p. 455].

Note that $(a, b, c)$ is determined by $(x, y)$ up to scalar multiplication, i.e., $(a, b, c)$ and $k(a, b, c)$ correspond to the same rational point in $\mathcal{D}$, so for any rational point $(x, y)$ one can choose $a, b, c$ to be integers with $a>0$.

A rational point in the interior of an edge $\langle p / q, r / s\rangle$ in $\mathcal{D}$ corresponds to a curve system $(1, b, c)$, which can be written as a linear combination

$$
(1, b, c)=\alpha(1, s-1, r)+\beta(1, q-1, p)
$$

where $\alpha, \beta$ are positive rational numbers, and $\alpha+\beta=1$. We write

$$
v=\alpha\langle r / s\rangle+\beta\langle p / q\rangle
$$

to indicate that the point $v$ is related to $\langle p / q\rangle$ and $\langle r / s\rangle$ as above. The number $\alpha$ (resp. $\beta$ ) is called the length of the edge segment from $\langle p / q\rangle$ (resp. $\langle r / s\rangle$ ) to $v$. It is important to note that this is not the euclidean length of the segments of the edge cut by $v$, even if the length of the edge is normalized to 1 . From the construction of the candidate surface ([4, p. 457]), we see that traveling from the vertex $\langle r / s\rangle$ to the point $v$ above corresponds to adding $m \beta$ saddles to the surface, where $m$ is the number of times the surface intersects a meridian of $K$, which must be an integer. This fact will be useful in the calculation of the Euler number of the resulting surface.

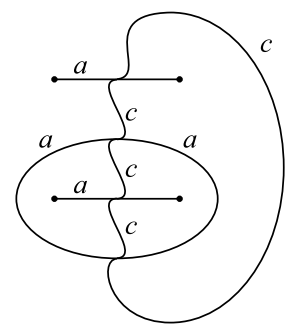

Figure 2: 
To make calculation easier, we introduce the $u$-coordinate of a point $v$. Define

$$
u=u(v)=\frac{1}{1-x},
$$

where $x$ is the $x$-coordinate of the point $v$ in $\mathcal{D}$. Thus, we have $x=(u-1) / u$. The $u$-coordinate has two important properties.

(1) The $u$-coordinate of a vertex $\langle p / q\rangle$ is $q$.

(2) The length of an edge segment is equal to its length in $u$-coordinate when the length of the edge is normalized to 1 , as shown in the following lemma.

Lemma 2.1. Let $v=\alpha\langle r / s\rangle+\beta\langle p / q\rangle$. Let $u=u(v), u_{0}=q$ and $u_{1}=s$ be the $u$-coordinates of $v,\langle p / q\rangle$ and $\langle r / s\rangle$ respectively. Then

$$
u=\alpha u_{1}+\beta u_{0}
$$

In particular, $\alpha$ and $\beta$ can be calculated by the following formulas:

$$
\begin{aligned}
& \alpha=\frac{u-u_{0}}{u_{1}-u_{0}}=\frac{u-q}{s-q}, \\
& \beta=\frac{u_{1}-u}{u_{1}-u_{0}}=\frac{s-u}{s-q} .
\end{aligned}
$$

Proof. Suppose $\langle p / q\rangle$ is represented by the curve system $\left(a_{0}, b_{0}, c_{0}\right)$, and $\langle r / s\rangle$ by $\left(a_{1}, b_{1}, c_{1}\right)$. By definition we may choose $\left(a_{1}, b_{1}, c_{1}\right)$ so that $a_{1}=$ $a_{0}$. Then the $x$-coordinates of these points are $x_{i}=b_{i} /\left(a_{i}+b_{i}\right)$, hence $u_{i}=$ $1 /(1-x)=\left(a_{i}+b_{i}\right) / a_{i}=1+\left(b_{i} / a_{i}\right)$ for $i=0,1$.

By definition $v=a\langle r / s\rangle+\beta\langle p / q\rangle$ is represented by $\left\langle\alpha a_{1}+\beta a_{0}, \alpha b_{1}+\right.$ $\left.\beta b_{0}, \alpha c_{1}+\beta c_{0}\right\rangle$. Using the facts that $\alpha+\beta=1$ and $a_{0}=a_{1}$, we can calculate the $u$-coordinate of $\alpha\langle r / s\rangle+\beta\langle p / q\rangle$ as follows

$$
\begin{aligned}
u & =1+\frac{\alpha b_{1}+\beta b_{0}}{\alpha a_{1}+\beta a_{0}}=1+\frac{\alpha b_{1}+\beta b_{0}}{a_{0}} \\
& =\alpha\left(1+\frac{b_{1}}{a_{1}}\right)+\beta\left(1+\frac{b_{0}}{a_{0}}\right) \\
& =\alpha u_{1}+\beta u_{0} .
\end{aligned}
$$


Definition 2.1. The length $|\gamma|$ of an edge path $\gamma$ in $\mathcal{D}$ is defined by counting the length of a full edge as 1 , and the length of a partial edge from $\langle r / s\rangle$ to $\alpha\langle r / s\rangle+\beta\langle p / q\rangle$ as $\beta$.

\subsection{Euler numbers of points, edge paths and surfaces}

The knot $K=K\left(t_{1}, t_{2}, t_{3}\right)$ in $S^{3}$ can be constructed as follows. Let $\left(B_{i}, T_{i}\right)$ be a rational tangle of slope $t_{i}=p_{i} / q_{i}$, where $B_{i}=D^{2} \times I$, and $T_{i}$ consists of two strings with endpoints on the vertical diameters of $D^{2} \times \partial I$. Gluing the end disks $D^{2} \times \partial I$ of the tangles in a cyclic way, we get a knot in a solid torus $V$, which can be trivially embedded in $S^{3}$ to produce the knot $K=K\left(t_{1}, t_{2}, t_{3}\right)$ in $S^{3}$.

Denote by $M_{i}$ the tangle space $B_{i}-\operatorname{Int} N\left(T_{i}\right)$. Let $E_{i}$ be a disk in $M_{i}$ separating the two arcs of $T_{i}$, and let $D_{i}=D_{i}^{1} \cup D_{i}^{2}$ be a pair of disks properly embedded in $M_{i}$ such that $D_{i}^{j}$ intersects the meridian of the $j$-th string of $T_{i}$ at a single point and is disjoint from the meridians of the other string.

Define a number $m_{i}$ as follows. If $\gamma_{i}$ is a not a constant path, let $m_{i}$ be the minimal positive integer such that $m_{i} \times\left|\gamma_{i}\right|$ is an integer. If $\gamma_{i}$ is a constant path on $L\left(p_{i} / q_{i}\right)$ at a point with $u$-coordinate $\bar{u}$, let $m_{i}$ be the smallest positive integer such that $m_{i} \bar{u} / q_{i}$ is an integer. Let $n=\operatorname{lcm}\left(m_{1}, m_{2}, m_{3}\right)$ be the least common multiple of $m_{1}, m_{2}, m_{3}$, and let $m$ be a multiple of $n$. A candidate surface is said to have $m$ sheets if it intersects the meridian of $K$ (and hence the meridian of each strand of the tangles) at $m$ points.

Lemma 2.2. Let $F\left(\gamma_{i}\right)$ be an m-sheet surface in the tangle space $M_{i}$ corresponding to the edge path $\gamma_{i}$ constructed in [4, p. 457].

(1) If $\gamma_{i}$ is not a constant path then $\chi\left(F\left(\gamma_{i}\right)\right)=m\left(2-\left|\gamma_{i}\right|\right)$.

(2) If $\gamma_{i}$ is a constant path on the horizontal edge $L\left(t_{i}\right)$ with u-coordinate $\bar{u}$, then $\chi\left(F\left(\gamma_{i}\right)\right)=m\left(1+\bar{u} / q_{i}\right)$

(3) Let $m_{i}$ be defined as above, and let $n=\operatorname{lcm}\left(m_{1}, m_{2}, m_{3}\right)$. Then there exists an $m$-sheet orientable candidate surface $F=F\left(\gamma_{1}, \gamma_{2}, \gamma_{3}\right)$ with $m=n$ or $2 n$.

Proof. (1) If $\gamma_{i}$ is not a constant path in the interior of $L\left(t_{i}\right)$ then according to [4, p. 457], $F\left(\gamma_{i}\right)$ is obtained from $m$ copies of $D_{i}$ by adding some saddles. For each full edge in $\gamma_{i}$ one adds $m$ saddles, and for a partial edge of length $\beta_{i}$ one adds $m \beta_{i}$ saddles. (By the choice of $m$ in the construction, $m \beta_{i}$ must be an integer.) Since $\chi\left(m D_{i}\right)=2 m$, and adding a saddle reduces the Euler characteristic by 1 , we have $\chi\left(F\left(\gamma_{i}\right)\right)=2 m-m\left|\gamma_{i}\right|$. 
(2) Suppose $\gamma_{i}$ is a constant path in the interior of the horizontal edge $L\left(t_{i}\right)$. Then by [4, p. 457], $F\left(\gamma_{i}\right)$ consists of $m$ copies of $D_{i}$ and $k$ copies of $E_{i}$ for some $k$, hence $\chi\left(F\left(\gamma_{i}\right)\right)=2 m+k$. We need to determine the number $k$.

Let $\left(a^{\prime}, b^{\prime}, c^{\prime}\right)$ be the parameters of $\partial D_{i}$ on the four-punctured sphere $\partial M_{i}$. Since it is a vertex on $L\left(t_{i}\right)$, we have $y_{i}=p_{i} / q_{i}=c^{\prime} /\left(a^{\prime}+b^{\prime}\right)$, and $x_{i}=\left(q_{i}-1\right) / q_{i}=b^{\prime} /\left(a^{\prime}+b^{\prime}\right)$. Also $a^{\prime}=1$ because a meridian intersects $\partial D_{i}$ at a single point. Solving these equations gives $b^{\prime}=q_{i}-1$, and $c^{\prime}=p_{i}$.

Let $\left(a^{\prime \prime}, b^{\prime \prime}, c^{\prime \prime}\right)$ be the parameters of $\partial E_{i}$. Then $a^{\prime \prime}=0$ because $\partial E_{i}$ is disjoint from the meridians of $T_{i}$. Examining the curve on $\partial B_{i}$ explicitly we see that $b^{\prime \prime}=q_{i}$ and $c^{\prime \prime}=p_{i}$, hence it has parameters $\left(0, q_{i}, p_{i}\right)$.

Now the parameters of $m D_{i}+k E_{i}$ are given by

$$
m\left(1, q_{i}-1, p_{i}\right)+k\left(0, q_{i}, p_{i}\right)=\left(m,(m+k) q_{i}-m,(m+k) p_{i}\right) .
$$

Hence the $x$-coordinate and $u$-coordinate of the constant path $\gamma_{i}$ satisfy

$$
\begin{aligned}
x_{i} & =\frac{(m+k) q_{i}-m}{(m+k) q_{i}}, \\
\bar{u} & =\frac{1}{1-x_{i}}=\frac{(m+k) q_{i}}{m}=q_{i}+\frac{k q_{i}}{m} .
\end{aligned}
$$

Solving the last equation gives $k=m \bar{u} / q_{i}-m$, therefore $\chi\left(F\left(\gamma_{i}\right)\right)=2 m+$ $k=m+m \bar{u} / q_{i}$.

(3) Let $F^{\prime}$ be an $n$-sheet candidate surface corresponding to the candidate system, constructed in [4, p. 457]. If $F^{\prime}$ is orientable then we are done. If not, let $F$ be the boundary of a regular neighborhood of $F^{\prime}$. Then $F$ is an orientable surface. It can be constructed by doubling the intersection of $F^{\prime}$ with each tangle space and therefore is a candidate surface corresponding to the same candidate system, with $m=2 n$ sheets.

Definition 2.2. Let $v$ be a rational point in $\mathcal{D}$ with $u=u(v)$ as its $u$-coordinate, and let $\gamma$ be an edge path with $v$ as its ending point.

(1) If $v$ is not on a horizontal line, define $e(v)=\frac{1}{3}(4-u(v))$.

(2) If $v$ is on a horizontal line $L(p / q)$, define $e(v)=\frac{1}{3}+u(v)\left(\frac{1}{q}-\frac{1}{3}\right)$.

(3) For an allowable edge path $\gamma$ with ending point $v$, define $e(\gamma)=e(v)-$ $|\gamma|$.

(4) Given a candidate edge path system $\left(\gamma_{1}, \gamma_{2}, \gamma_{3}\right)$, define $\bar{e}=$ $\bar{e}\left(\gamma_{1}, \gamma_{2}, \gamma_{3}\right)=\sum e\left(\gamma_{i}\right)$. 
The numbers $e(v), e(\gamma)$ and $\bar{e}$ are called the Euler number of a point, an edge path, and a candidate system, respectively.

Example 2.1. (a) If $v \in L(p / 2)$ then $e(v)=\frac{1}{3}+\frac{1}{6} u>0$.

(b) If $v \in L(p / 3)$ then $e(v)=\frac{1}{3}>0$.

(c) If $v \in L(p / q)$ and $q \geq 4$ then $e(v)=\frac{1}{3}+u\left(\frac{1}{q}-\frac{1}{3}\right) \leq \frac{1}{3}+q\left(\frac{1}{q}-\frac{1}{3}\right)=$ $\frac{4}{3}-\frac{q}{3} \leq 0$, and $e(v)=0$ if and only if $q=u=4$.

(d) At a vertex $v=\langle p / q\rangle, u(v)=q$, so $e(v)$ can be rewritten as $e(v)=$ $\frac{1}{3}(4-q)$; in particular, $e(\langle p / q\rangle)<0$ for all $q>4$.

Lemma 2.3. Let $\gamma$ be an edge path with $|\gamma|<1$. Let $v$ be the ending point of $\gamma$, and let $u=u(v)$ be the $u$-coordinate of $v$. Then

(1) $e(\gamma)>0$ if and only if $(i) v$ is on the horizontal edge $L(p / q)$ with $q \leq 3$, (ii) $v$ is on $\langle p / 1, r / s\rangle$ for some $s \leq 3$, or (iii) $v$ is on $\langle p / 2, r / 3\rangle$ and $u>2.5$.

(2) $e(\gamma)=0$ if and only if $(i) v$ is on $\langle p / 1, r / 4\rangle$, or (ii) $v$ is on $\langle p / 2, r / 3\rangle$ and $u=2.5$.

Proof. Since $|\gamma|<1, \gamma$ cannot contain a full edge, hence if $v$ is a vertex then $\gamma$ is a constant path. By definition $\gamma$ is also a constant path if $v$ is in the interior of a horizontal edge. Thus if $v$ is on a horizontal line $L(p / q)$ then the result follows from the calculations in Example 2.1(4) because $e(\gamma)=e(v)$.

We now assume that $v \in\langle p / q, r / s\rangle$, and $v \neq\langle p / q\rangle,\langle r / s\rangle$. Let $\bar{u}$ be the $u$ coordinate of $v$. Then by definition we have $e(\gamma)=\frac{1}{3}(4-\bar{u})-(s-\bar{u}) /(s-$ $q)$, which is a linear function of $\bar{u}, e(\gamma)=e(\langle p / q\rangle)-1$ when $\bar{u}=q$, and $e(\gamma)=e(\langle r / s\rangle)$ when $\bar{u}=s$. Thus when $s \geq 5$ we have $e(\gamma) \leq 0$ at $\bar{u}=q$, and $<0$ at $\bar{u}=s$, hence $e(\gamma)<0$ for all $q<\bar{u} \leq s$. The cases where $1 \leq q<s \leq 4$ can be done one by one. We omit the details.

The set of $v$ such that $e(\gamma)>0$ for some $\gamma$ ending at $v$ is shown in figure 3 for the square $[0,1] \times[0,1]$. Those in the other squares are vertical translations of this graph.

Theorem 2.1. Let $K=K\left(p_{1} / q_{1}, p_{2} / q_{2}, p_{3} / q_{3}\right)$ be a Montesinos knot of length 3 , let $\left(\gamma_{1}, \gamma_{2}, \gamma_{3}\right)$ be a candidate system, let $F=F\left(\gamma_{1}, \gamma_{2}, \gamma_{3}\right)$ be the associated candidate surface, and let $\hat{F}=\hat{F}\left(\gamma_{1}, \gamma_{2}, \gamma_{3}\right)$ be the corresponding closed surface. Denote by $r=a / b$ the boundary slope of $F$, where $a, b$ are coprime integers. Then $\hat{F}$ is a torus if and only if

$$
\bar{e}=\sum e\left(\gamma_{i}\right)=\frac{b-1}{b} .
$$




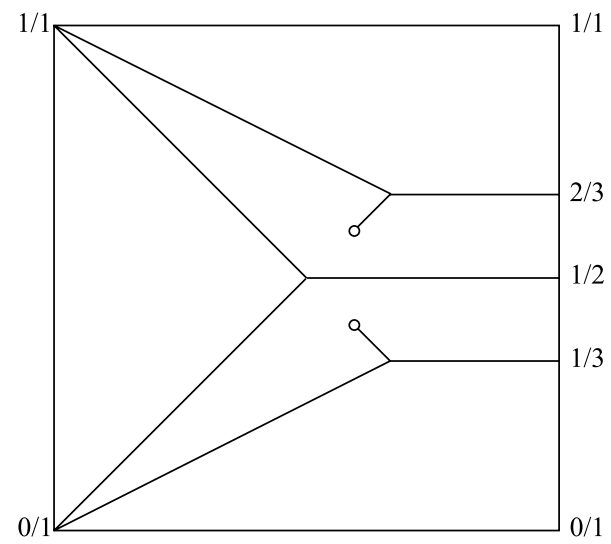

Figure 3:

In particular, if $r$ is an integer slope then $\bar{e}=0$, and if $r$ is a half integer slope then $\bar{e}=\frac{1}{2}$.

Proof. Let $\left(a_{i}, b_{i}, c_{i}\right)$ be the parameters of the ending point of $\gamma_{i}$, chosen so that $a_{1}=a_{2}=a_{3}$ for all $i$, which will be denoted by $m$. Since $x_{i}=b_{i} /\left(b_{i}+\right.$ $a_{i}$ ) are the same for a candidate system, we have $b_{1}=b_{2}=b_{3}$, which we denote by $b$.

First consider the surface $F^{\prime}$ obtained by gluing $F\left(\gamma_{i}\right)$ along the three twice punctured disks $P_{j}$ on the boundary of the tangle spaces. Each $F\left(\gamma_{i}\right)$ intersects $P_{j}$ at $2 m+b$ arcs, hence after gluing the $F\left(\gamma_{i}\right)$ to each other, we have

$$
\chi\left(F^{\prime}\right)=\sum \chi\left(F\left(\gamma_{i}\right)\right)-3(2 m+b)
$$

By construction $F=F\left(\gamma_{1}, \gamma_{2}, \gamma_{3}\right)$ is obtained from $F^{\prime}$ by adding $2 m+2 b$ disjoint meridional disks in the solid torus $S^{3}-\cup B_{i}$, hence

$$
\chi(F)=\chi\left(F^{\prime}\right)+2 m+2 b=\sum \chi\left(F\left(\gamma_{i}\right)\right)-4 m-b .
$$

From $x=b_{i} /\left(a_{i}+b_{i}\right)=b /(m+b)$ and $\bar{u}=1 /(1-x)$, one can solve $b$ to obtain

$$
b=\frac{m x}{1-x}=m(\bar{u}-1)
$$


When $r$ is an integer slope, we need to attach $m$ disks to $F$ to obtain $\hat{F}$, hence

$$
\begin{aligned}
\chi(\hat{F}) & =\chi(F)+m=\sum \chi\left(F\left(\gamma_{i}\right)\right)-3 m-m(\bar{u}-1) \\
& =m \sum\left(\frac{1}{m} \chi\left(F\left(\gamma_{i}\right)\right)-\frac{2}{3}-\frac{1}{3} \bar{u}\right) .
\end{aligned}
$$

If $\gamma_{i}$ is a constant path, by Lemma 2.2(2) and Definition 2.2 we have

$$
\begin{aligned}
\frac{1}{m} \chi\left(F\left(\gamma_{i}\right)\right)-\frac{2}{3}-\frac{1}{3} \bar{u} & =\left(1+\frac{\bar{u}}{q_{i}}\right)-\frac{2}{3}-\frac{1}{3} \bar{u} \\
& =\frac{1}{3}+\bar{u}\left(\frac{1}{q_{i}}-\frac{1}{3}\right)=e\left(\gamma_{i}\right)
\end{aligned}
$$

If $\gamma_{i}$ is not a constant path, by Lemma 2.2(1) and Definition 2.2 we have

$$
\begin{aligned}
\frac{1}{m} \chi\left(F\left(\gamma_{i}\right)\right)-\frac{2}{3}-\frac{1}{3} \bar{u} & =\left(2-\left|\gamma_{i}\right|\right)-\frac{2}{3}-\frac{1}{3} \bar{u} \\
& =\frac{4}{3}-\frac{1}{3} \bar{u}-\left|\gamma_{i}\right|=e\left(\gamma_{i}\right)
\end{aligned}
$$

Therefore, we always have $\chi(\hat{F})=m \sum e\left(\gamma_{i}\right)$, hence $\hat{F}$ is a torus if and only if $\sum e\left(\gamma_{i}\right)=0$.

The proof for $r=a / b$ and $b \neq 1$ is similar. In this case $F$ has $m / b$ boundary components, so $\hat{F}$ is obtained by attaching $m / b$ disks to $F$. Hence a similar calculation shows that

$$
\chi(\hat{F})=\chi(F)+\frac{m}{b}=(\chi(F)+m)-\frac{b-1}{b} m=m\left(\sum e\left(\gamma_{i}\right)-\frac{b-1}{b}\right) .
$$

Therefore in this case $\hat{F}$ is a torus if and only if $\sum e\left(\gamma_{i}\right)=(b-1) / b$.

Proposition 2.2. Let $\left(\gamma_{1}, \gamma_{2}, \gamma_{3}\right)$ be a candidate system such that $\hat{F}\left(\gamma_{1}, \gamma_{2}\right.$, $\gamma_{3}$ ) is a torus. Suppose $\bar{u} \leq 1$. Then (i) $\bar{u}=1$, and (ii) $K=K\left(1 / q_{1}, 1 / q_{2}\right.$, $1 / q_{3}$ ) for some (possibly negative) integers $q_{i}$, such that $\left|q_{i}\right|>1$, and at most one $q_{i}$ is even.

The knots and the corresponding toroidal slopes are the same as those in Theorem 1.1(1) and (2).

Proof. Let $\gamma_{i}^{\prime}$ be the part of $\gamma_{i}$ in the strip of $x \in[0,1$ ) (i.e., $u \geq 1$ ), and let $y_{i}^{\prime}$ be the ending points of $\gamma_{i}^{\prime}$. Then $\left|\gamma_{i}^{\prime}\right|$ are all nonzero integers, hence 
we have

$$
0 \leq \bar{e}=(4-\bar{u})-\sum\left|\gamma_{i}\right| \leq 3-\sum\left|\gamma_{i}^{\prime}\right| \leq 0
$$

Thus all the inequalities above are equalities, and we have $\bar{u}=\left|\gamma_{i}\right|=\left|\gamma_{i}^{\prime}\right|=1$ for all $i$, so $\gamma_{i}=\gamma_{i}^{\prime}$ contains only one edge. Since $\bar{u}=1, y_{i}=y_{i}^{\prime}$ are integers. By definition of candidate system we have $\sum y_{i}=0$, hence by choosing the parameters properly we may assume that $y_{i}=0$ for all $i$. It is now easy to see that $K=K\left(1 / q_{1}, 1 / q_{2}, 1 / q_{3}\right)$ for some $q_{i}$. Since $K$ is of length $3,\left|q_{i}\right|>1$. Since $K$ is a knot, at most one $q_{i}$ is even.

The knots are the same as those in Theorem 1.1(1) and (2). The toroidal surface corresponding to a candidate system above is the pretzel surface $S$, or its double cover if $S$ is nonorientable. One can draw the pretzel surface and show that the boundary slope of $F$ is the same as that in Theorem $1.1(1)$ and (2).

Up to equivalence we may change the parameters of $K=K\left(t_{1}, t_{2}, t_{3}\right)$ by the following moves.

(1) Replace all $t_{i}$ by $-t_{i}$;

(2) Permute $t_{i}$;

(3) Replace $\left(t_{1}, t_{2}, t_{3}\right)$ by $\left(t_{1}+k_{1}, t_{2}+k_{2}, t_{3}+k_{3}\right)$, where $k_{i}$ are integers, and $\sum k_{i}=0$.

If $\left(\gamma_{1}, \gamma_{2}, \gamma_{3}\right)$ is a candidate system for $K\left(t_{1}, t_{2}, t_{3}\right)$, and $\left(t_{1}^{\prime}, t_{2}^{\prime}, t_{3}^{\prime}\right)$ is equivalent to $\left(t_{1}, t_{2}, t_{3}\right)$ by the above relations, then we can obtain a candidate system $\left(\gamma_{1}^{\prime}, \gamma_{2}^{\prime}, \gamma_{3}^{\prime}\right)$ for $K\left(t_{1}^{\prime}, t_{2}^{\prime}, t_{3}^{\prime}\right)$ in the obvious way. For example, when $\left(t_{1}, t_{2}, t_{3}\right)$ is replaced by $\left(t_{1}+1, t_{2}-1, t_{3}\right)$, the edge path $\gamma_{1}^{\prime}$ is obtained by moving $\gamma_{1}$ upward by one unit, and $\gamma_{2}^{\prime}$ downward by one unit. Clearly the surface $F\left(\gamma_{1}, \gamma_{2}, \gamma_{3}\right)$ is homeomorphic to $F\left(\gamma_{1}^{\prime}, \gamma_{2}^{\prime}, \gamma_{3}^{\prime}\right)$.

Let $G=\left\langle 0,-\frac{1}{3}\right\rangle \cup\left\langle 0,-\frac{1}{2}\right\rangle \cup\left\langle-\frac{1}{2},-\frac{1}{3}\right\rangle \cup\left\langle-\frac{1}{2},-\frac{2}{3}\right\rangle \cup\left\langle-1,-\frac{1}{2}\right\rangle \cup L\left(-\frac{1}{2}\right) \cup$ $L\left(-\frac{1}{3}\right)$, as shown in figure 4 .

Lemma 2.4. Let $\left(\gamma_{1}, \gamma_{2}, \gamma_{3}\right)$ be a candidate edge path system for $K=$ $K\left(t_{1}, t_{2}, t_{3}\right)$ such that the corresponding surface $\hat{F}\left(\gamma_{1}, \gamma_{2}, \gamma_{3}\right)$ is a torus. Let $v_{i}=\left(x_{i}, y_{i}\right)$ be the ending point of $\gamma_{i}$, and let $\bar{u}$ be the u-coordinate of $v_{i}$. Assume $\bar{u}>1$. Then the following hold up to re-choosing the parameters of $K$.

(1) $\sum y_{i}=0$;

(2) $\left|y_{i}\right|+\left|y_{j}\right| \leq 1$ for any $i \neq j$; 


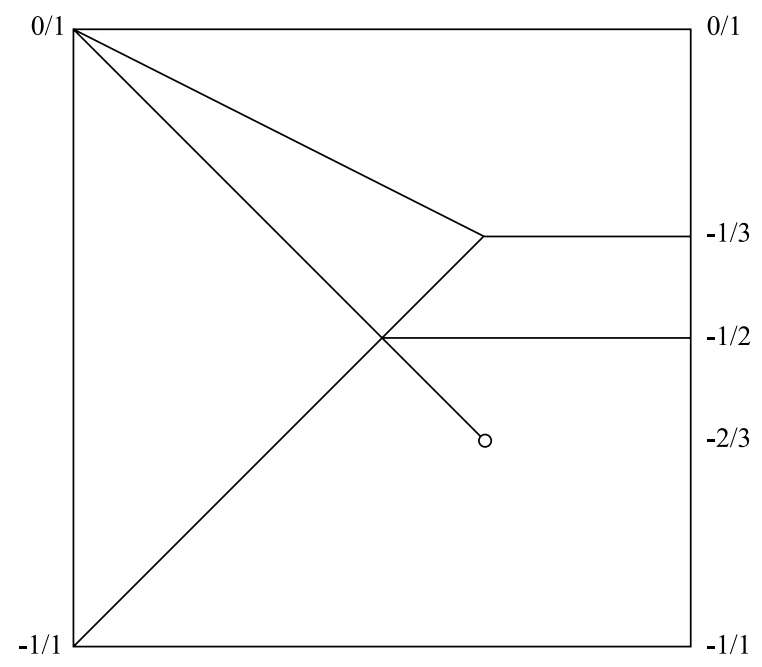

Figure 4:

(3) $0<\left|y_{i}\right| \leq \frac{2}{3}$;

(4) $v_{1}$ is on the subgraph $G$ in figure 4.

Proof. We may assume that the parameters of the knot has been chosen, among equivalent knots, so that $\sum\left|y_{i}\right|$ is minimal. The minimum can be reached because (i) $\sum\left|y_{i}\right|$ remains the same when permuting the parameters or replacing $\left(t_{1}, t_{2}, t_{3}\right)$ by $\left(-t_{1},-t_{2},-t_{3}\right)$, and (ii) $\sum\left|y_{i}\right|$ goes to $\infty$ when $\left(t_{1}, t_{2}, t_{3}\right)$ is replaced by $\left(t_{1}+k_{1}, t_{2}+k_{2}, t_{3}-k_{1}-k_{2}\right)$ and at least one $k_{i}$ goes to $\infty$.

(1) This follows from the definition of candidate system.

(2) By permuting the $t_{i}$ and simultaneously changing their signs if necessary, we may assume without loss of generality that $-y_{1} \geq y_{2} \geq y_{3} \geq 0$. If the result is false then $-y_{1}+y_{2}>1$. But then replacing $\left(t_{1}, t_{2}, t_{3}\right)$ of $K$ by $\left(t_{1}+1, t_{2}-1, t_{3}\right)$ will give a candidate system such that the $y$-coordinates of the ending points are $y_{1}^{\prime}=-y_{1}+1, y_{2}^{\prime}=y_{2}-1$, and $y_{3}^{\prime}=y_{3}$, respectively. One can check that $\sum\left|y_{i}^{\prime}\right|<\sum\left|y_{i}\right|$ if $\left|y_{1}\right|+\left|y_{2}\right|>1$.

(3) Since $\bar{u}>1, v_{i}$ cannot be on $L(0)$ as otherwise $\gamma_{i}$ would be a constant path on $L(0)$, so $t_{i}$ would be 0 , contradicting the assumption that the parameters of $K=K\left(t_{1}, t_{2}, t_{3}\right)$ are non-integers. Therefore, $y_{i} \neq 0$. If $\left|y_{1}\right|>\frac{2}{3}$, say, then since $y_{1}=-y_{2}-y_{3}$, we would have $\left|y_{i}\right|>\frac{1}{3}$ for $i=2$ or 3 , which implies $\left|y_{1}\right|+\left|y_{i}\right|>1$, contradicting (2).

(4) Up to relabeling we may assume that $e\left(\gamma_{1}\right) \geq e\left(\gamma_{i}\right)$ for $i=2,3$, and by taking the mirror image of $K$ if necessary we may assume that $y_{1}<0$. 
By Theorem 2.1, $\bar{e}=\sum e\left(\gamma_{i}\right) \geq 0$, hence either $e\left(\gamma_{1}\right)>0$, or $e\left(\gamma_{i}\right)=0$ for all $i$.

First assume $e\left(\gamma_{1}\right)>0$. Since $-1<y_{1}<0$, by Lemma $2.3 v_{1}$ is on one of the edges in $G$, except that it may also be on the edge $L\left(-\frac{2}{3}\right)$. However, if $v_{1} \in L\left(-\frac{2}{3}\right)$ then since $-y_{1}=y_{2}+y_{3}$ and $-y_{1}+y_{i} \leq 1$, we must have $y_{2}=y_{3}=\frac{1}{3}$, hence replacing $\left(t_{1}, t_{2}, t_{3}\right)$ by $\left(-t_{2},-t_{3},-t_{1}\right)$ will give a new candidate system such that the ending point of the first edge path is on $L\left(-\frac{1}{3}\right)$, as required.

Now assume $e\left(\gamma_{i}\right)=0$ for all $i$. We may assume that no $v_{i}$ is on $G$ or its reflection along the line $y=0$, as otherwise we may choose the parameters of $K$ so that $v_{1} \in G$. Thus by Lemmas 2.3 and 2.4(3), each $v_{i}$ must be on $\left\langle 0, \pm \frac{1}{4}\right\rangle$. However, in this case one can show that $\sum y_{i} \neq 0$, contradicting Lemma 2.4(1). Therefore this case cannot happen.

\subsection{Calculation of boundary slopes}

Denote by $e_{-}\left(\right.$resp. $\left.e_{+}\right)$the number of edges in all the $\gamma_{i}$ on which a point moves downward (resp. upward) when traveling from right to left. Then the twist number of the edge path system $\left(\gamma_{1}, \gamma_{2}, \gamma_{3}\right)$ is defined as

$$
\tau=\tau\left(\gamma_{1}, \gamma_{2}, \gamma_{3}\right)=2\left(e_{-}-e_{+}\right) .
$$

Denote by $\delta=\delta\left(\gamma_{1}, \gamma_{2}, \gamma_{3}\right)$ the boundary slope of the surface $F\left(\gamma_{1}, \gamma_{2}, \gamma_{3}\right)$. The following lemma is due to Hatcher and Oertel [4], and can be used to calculate the boundary slope $\delta$ for a given edge path system.

Lemma 2.5. Let $t_{i}$ be rational numbers, and let $\left(\gamma_{1}, \gamma_{2}, \gamma_{3}\right)$ be a candidate system with $\gamma_{i}$ starting at a point on $L\left(t_{i}\right)$. Then $\delta-\tau$ depends only on $t_{i}$ and is independent of the paths $\gamma_{i}$.

Thus, if $F^{\prime}=F\left(\gamma_{1}^{\prime}, \gamma_{2}^{\prime}, \gamma_{3}^{\prime}\right)$ has boundary slope $\delta^{\prime}$ and $\gamma_{i}^{\prime}$ has starting point on $L\left(t_{i}\right)$, then $\delta=\tau+\delta^{\prime}-\tau^{\prime}$, where $\delta^{\prime}=\delta\left(\gamma_{1}^{\prime}, \gamma_{2}^{\prime}, \gamma_{3}^{\prime}\right)$ and $\tau^{\prime}=\tau\left(\gamma_{1}^{\prime}, \gamma_{2}^{\prime}, \gamma_{3}^{\prime}\right)$. In particular if $F^{\prime}$ is a Seifert surface then $\delta=\tau-\tau^{\prime}$.

Proof. This is in page 460 of [4], where it was shown that $\delta=\tau-\tau^{\prime}$, where $\tau^{\prime}$ is the twist number of the edge path system corresponding to the Seifert surface of $K$, starting from the vertices $\left\langle t_{i}\right\rangle$. Therefore $\delta-\tau$ depends only on $\left(t_{1}, t_{2}, t_{3}\right)$. 


\subsection{Notations and conventions}

Throughout this paper we will denote by $\gamma_{i}$ the edge path for the $i$-th tangle, by $v_{i}$ the ending point of $\gamma_{i}$, by $\bar{u}$ the $u$-coordinate of $v_{i}$, which must be the same for all $i$, and by $y_{i}$ the $y$-coordinate of $v_{i}$. Let $L$ be the union of the two horizontal edges in $G$, i.e., $L=L(-1 / 2) \cup L(-1 / 3)$.

The case $\bar{u} \leq 1$ has been discussed in Proposition 2.2. Hence in Sections 3-6 we will assume that $\bar{u}>1$. By Lemma 2.4, in this case we may choose the parameters $t_{i}$ of $K=K\left(t_{1}, t_{2}, t_{3}\right)$ to satisfy the conclusions of that lemma; in particular, the ending point $v_{1}$ of $\gamma_{1}$ lies on the subgraph $G$ of $\mathcal{D}$ in figure 4 . In Sections 3-6 we will determine $K$ case by case, according to the position of $v_{1}$ in $G$.

\section{The case that $v_{1} \in L$ and $\alpha_{i}=0$ for $i=2$ or 3}

In this section, we will discuss the case that one of the vertices, say $v_{1}$, lies on the horizontal lines $L=L(-1 / 2) \cup L(-1 / 3)$, and $\alpha_{2}=0$. Note that the second condition is equivalent to that either $\gamma_{2}$ is a constant path, or $v_{2}$ is a vertex of $\mathcal{D}$.

Lemma 3.1. $\gamma_{i}$ cannot all be constant paths.

Proof. Let $y_{i}=p_{i} / q_{i}$ be the $y$-coordinates of the ending points $v_{i}$ of $\gamma_{i}$, where $p_{i}, q_{i}$ are coprime integers. By Lemma 2.4(1) we have

$$
\sum y_{i}=\sum \frac{p_{i}}{q_{i}}=0
$$

If all $\gamma_{i}$ are constant paths, $K=K\left(p_{1} / q_{1}, p_{2} / q_{2}, p_{3} / q_{3}\right)$ and since $K$ is a knot, at most one of the $q_{i}$ is even. If one of the $q_{i}$, say $q_{1}$, is even, then we have

$$
q_{2} q_{3} p_{1}+q_{1}\left(q_{3} p_{2}+q_{2} p_{3}\right)=0 .
$$

Since the first term is odd and the other two are even, this is impossible. If all $q_{i}$ are odd, then equation (3.1) implies that

$$
q_{2} q_{3} p_{1}+q_{1} q_{3} p_{2}+q_{1} q_{2} p_{3} \equiv p_{1}+p_{2}+p_{3} \equiv 0 \quad \bmod 2
$$

which implies that either one or three $p_{i}$ are even. However, in this case $K$ is a link of two components, which is again a contradiction. 
Lemma 3.2. If $v_{1}$ is in the interior of $L$, and $v_{2}$ is in the interior of $L\left(p_{2} / q_{2}\right)$ for some $q_{2} \leq 3$, then $K=K(-1 / 2,1 / 3,1 /(6+1 / n))$ for some $n \neq 0,-1$, and $\bar{u}=6$.

Proof. Recall that we have assumed that $y_{i}$ satisfy the conclusions of Lemma 2.4, hence $y_{1}+y_{2}+y_{3}=0,0<\left|y_{i}\right| \leq \frac{2}{3}$, and $\left|y_{i}\right|+\left|y_{j}\right| \leq 1$ for $i \neq j$.

First assume $y_{1}=-\frac{1}{2}$. Then the above and the assumption of $v_{2} \in$ $L\left(p_{2} / q_{2}\right)$ for $q_{2} \leq 3$ imply that $y_{2}=\frac{1}{3}$, and $y_{3}=-y_{1}-y_{2}=1 / 6$. The horizontal line $y=1 / 6$ intersects the graph $\mathcal{D}$ at the horizontal edge $L(1 / 6)$ and one point on each edge $\langle 0,1 / q\rangle$ with $q \leq 6$. By Lemma 3.1, $v_{3}$ cannot be in the interior of $L(1 / 6)$ as otherwise we would have three constant paths. It follows that $v_{3}$ must be on some $\langle 0,1 / q\rangle$ with $q \leq 6$. By calculating the intersection point of $y=1 / 6$ with $\langle 0,1 / q\rangle$ we see that $u \leq 3$ when $q \leq 5$, which would be a contradiction because $v_{2} \in \operatorname{Int} L\left(\frac{1}{3}\right)$ implies that $\bar{u}>3$. Therefore, we must have $q=6$, in which case $v_{3}$ is the vertex $\langle 1 / 6\rangle$.

By Definition 2.2 we have

$$
\sum e\left(v_{i}\right)=\left(\frac{1}{3}+6\left(\frac{1}{2}-\frac{1}{3}\right)\right)+\frac{1}{3}+\frac{1}{3}(4-6)=1,
$$

therefore by Theorem 2.1 we must have $\bar{e}=\sum e\left(\gamma_{i}\right)=\sum e\left(v_{i}\right)-\sum\left|\gamma_{i}\right|=0$, so there is exactly one edge in $\cup \gamma_{i}$, which must be in $\gamma_{3}$ because $\gamma_{1}$ and $\gamma_{2}$ are constant paths. Therefore

$$
K=K\left(-\frac{1}{2}, \frac{1}{3}, \frac{1}{6+\frac{1}{n}}\right) .
$$

Since $\gamma_{3}$ must be an allowable edge path, we have $n \neq 0,-1$, and the result follows.

Now assume $y_{1}=-\frac{1}{3}$. The case of $y_{2}=\frac{1}{2}$ is similar to the above, and we obtain the same knot up to equivalence. If $y_{2}=-\frac{1}{2}$ then $\left|y_{2}\right|+\left|y_{3}\right|>1$, contradicting Lemma 2.4. In all other cases we have $v_{i} \in \operatorname{Int} L\left(p_{i} / 3\right)$ for each $i$, which implies that $\gamma_{i}$ are all constant paths, contradicting Lemma 3.1.

Lemma 3.3. Suppose $v_{1} \in L(-1 / 3)$. Then $v_{2}$ cannot be in the interior of a horizontal edge $L\left(p_{2} / q_{2}\right)$ with $q_{2} \geq 4$.

Proof. If this is not true then $\gamma_{2}$ is a constant path, so by Lemma $3.1 \gamma_{3}$ cannot be a constant path, hence $\left|\gamma_{3}\right|>0$. By Definition 2.2 we have

$$
e\left(\gamma_{1}\right)=\frac{1}{3}
$$




$$
\begin{aligned}
e\left(\gamma_{2}\right) & =\frac{1}{3}+\bar{u}\left(\frac{1}{q_{2}}-\frac{1}{3}\right) \\
e\left(\gamma_{3}\right) & =\frac{1}{3}(4-\bar{u})-\left|\gamma_{3}\right| \\
0 \leq \bar{e} & =\sum e\left(\gamma_{i}\right)=2-\frac{5}{12} \bar{u}-\bar{u}\left(\frac{1}{4}-\frac{1}{q_{2}}\right)-\left|\gamma_{3}\right| \\
& \leq 2-\frac{5}{12} \bar{u}-\left|\gamma_{3}\right|<2-\frac{5}{12} \bar{u} .
\end{aligned}
$$

This gives $5>\bar{u}$. Since $\bar{u} \geq q_{3}$ and $q_{3} \geq 4$, we must have $q_{2}=4$. Assume $v_{3}$ is on the edge $\left\langle p_{3} / q_{3}, t_{3} / s_{3}\right\rangle$. Then $s_{3}>\bar{u} \geq 4$, so $s_{3} \geq 5$.

Define $\beta_{3}(u)=\left(s_{3}-u\right) /\left(s_{3}-q_{3}\right)$. Then $\beta_{3}(\bar{u})$ is the length of the last edge segment in $\gamma_{3}$, so $\beta_{3}(\bar{u}) \leq\left|\gamma_{3}\right|$.

The function $e(u)=2-\frac{5}{12} u-\beta_{3}(u)$ is a linear function of $u$. We have $e(5)<0$, and $e(\bar{u}) \geq \bar{e} \geq 0$, for some $4<\bar{u}<5$, so $e(4)>0$, and hence $\beta_{3}(4)$ $<\frac{1}{3}$. Since $\beta_{3}(4)=\left(s_{3}-4\right) /\left(s_{3}-q_{3}\right)$, this is true if and only if $q_{3}=1$ and $s_{3}=5$. Hence $v_{3}$ is on an edge $E_{3}=\left\langle p_{3} / 1, t_{3} / 5\right\rangle$ for some $p_{3}, t_{3}$. Since $0<$ $\left|y_{3}\right| \leq \frac{2}{3}$, we must have $E_{3}=\langle 0, \pm 1 / 5\rangle$.

By assumption we have $y_{1}=-\frac{1}{3}$. Since $\left|y_{2}\right|=\left|p_{2} / q_{2}\right|=\left|p_{2}\right| / 4$ and $\left|y_{1}\right|+$ $\left|y_{2}\right| \leq 1$, we must have $\left|y_{2}\right|=\frac{1}{4}$, hence $\left(y_{2}, y_{3}\right)=\left(-\frac{1}{4}, \frac{5}{12}\right)$ or $\left(\frac{1}{4}, \frac{1}{12}\right)$. It is easy to see that the horizontal line $y=\frac{5}{12}$ does not intersect the edge $E_{3}$ above. Therefore, we must have $\left(y_{2}, y_{3}\right)=\left(\frac{1}{4}, \frac{1}{12}\right)$, hence $E_{3}=\left\langle 0, \frac{1}{5}\right\rangle$.

The line equation of $E_{3}$ is given by $y=x / 4$, hence the only solution for $y_{3}=1 / 12$ and $x>0$ is at $x=1 / 3$, which has $u$-coordinate $u=1 /(1-x)=$ $3 / 2<4$. Therefore, there is no solution in this case because $\bar{u}>4$.

Lemma 3.4. Suppose $v_{1} \in L\left(-\frac{1}{2}\right)$. Then $v_{2}$ cannot be in the interior of a horizontal edge $L\left(p_{2} / q_{2}\right)$ with $q_{2} \geq 4$.

Proof. Similar to Lemma 3.3, we have

$$
\begin{aligned}
& e\left(\gamma_{1}\right)=\frac{1}{3}+\bar{u}\left(\frac{1}{2}-\frac{1}{3}\right)=\frac{1}{3}+\frac{1}{6} \bar{u}, \\
& e\left(\gamma_{2}\right)=\frac{1}{3}+\bar{u}\left(\frac{1}{q_{2}}-\frac{1}{3}\right) \\
& e\left(\gamma_{3}\right)=\frac{1}{3}(4-\bar{u})-\left|\gamma_{3}\right|, \\
& 0 \leq \bar{e}=\sum e\left(\gamma_{i}\right)=2-\bar{u}\left(\frac{1}{2}-\frac{1}{q_{2}}\right)-\left|\gamma_{3}\right| .
\end{aligned}
$$


Since $K$ is a knot, $q_{2}$ must be odd, hence $q_{2} \geq 5$. Hence from the above we have $2-\bar{u}\left(\frac{1}{2}-\frac{1}{5}\right) \geq 0$, so $\bar{u}<7$. Since $q_{2}<\bar{u}$, we must have $q_{2}=5$. Therefore, $y_{2}=p_{2} / q_{2}= \pm k / 5$ for some $k=1,2,3,4$. Since $\left|y_{i}\right|+\left|y_{j}\right| \leq 1$ and $\left|y_{1}\right|=\frac{1}{2}$, we must have $y_{2}=\frac{1}{5}$ or $\frac{2}{5}$. (The cases of $y_{2}=-\frac{1}{5}$ and $-\frac{2}{5}$ are impossible because then $y_{3}>\frac{1}{2}$, so $\left|y_{1}\right|+\left|y_{3}\right|>1$.)

When $y_{2}=\frac{2}{5}$, we have $y_{3}=-y_{1}-y_{2}=\frac{1}{10}$. The intersection of the line $y=\frac{1}{10}$ and $\mathcal{D}$ is the union of $L\left(\frac{1}{10}\right)$ and one point in each edge $\left\langle 0,1 / q_{3}\right\rangle$ for $q_{3} \leq 10$. Since $\bar{u}<7, v_{2}$ cannot be on $L\left(\frac{1}{10}\right)$. By direct calculation we see that the $u$ value of the intersection between $y=\frac{1}{10}$ and $\left\langle 0,1 / q_{3}\right\rangle$ is

$$
u=\frac{1}{1-\left(q_{3}-1\right) y}=\frac{10}{11-q_{3}},
$$

which gives $u \leq 5$ for $q_{3} \leq 9$, and $u=10$ for $q_{3}=10$. Since $5<\bar{u}<7$, there is no solution in this case.

When $y_{2}=\frac{1}{5}$, we have $y_{3}=-y_{1}-y_{2}=\frac{3}{10}$. The horizontal line $y=\frac{3}{10}$ intersects $\mathcal{D}$ at $L\left(\frac{3}{10}\right)$, and one point on each of $\left\langle 0, \frac{1}{2}\right\rangle,\left\langle 0, \frac{1}{3}\right\rangle,\left\langle\frac{1}{3}, \frac{1}{4}\right\rangle$, and $\left\langle\frac{1}{3}, \frac{2}{7}\right\rangle$. (These are all the edges $\left\langle t_{1}, t_{2}\right\rangle$ with $t_{1}$ and $t_{2}$ on opposite sides of $y=\frac{3}{10}$.) As above, one can calculate the $u$-coordinate of the intersection to show that there is no intersection point on the interval $5<u<7$. Hence there is no solution in this case either.

We now assume that $v_{1} \in L$, and $v_{2}, v_{3}$ are not in the interior of horizontal edges. Since $\alpha_{2}=0$, the ending point $v_{2}$ of $\gamma_{2}$ must be a vertex of $\mathcal{D}$. The following two lemmas determine all knots with this property.

Lemma 3.5. Suppose $v_{1} \in L(-1 / 3), v_{2}$ is a vertex of $\mathcal{D}$, and $v_{3}$ is not in the interior of a horizontal edge. Then $K=K(-1 / 3,-1 /(3+1 / n), 2 / 3)$ for some odd $n \neq-1$, and $\bar{u}=3$.

Proof. By Definition 2.2 we have

$$
0 \leq \bar{e}=\sum e\left(\gamma_{i}\right) \leq \frac{1}{3}+2 \times \frac{1}{3}(4-\bar{u})-\sum\left|\gamma_{i}\right|,
$$

which gives $\bar{u} \leq 4.5$. Since $v_{2}$ is vertex and $v_{1} \in L(-1 / 3), \bar{u}$ is an integer, and $\bar{u} \geq 3$. Hence $\bar{u}=3$ or 4 . Thus $v_{2}=\langle \pm 1 / 3\rangle,\langle \pm 2 / 3\rangle,\langle \pm 1 / 4\rangle$ or $\langle \pm 3 / 4\rangle$. Since $\left|y_{i}\right| \leq \frac{2}{3}$, we cannot have $\left|y_{2}\right|=3 / 4$.

When $y_{2}=-1 / 3$, we have $y_{3}=2 / 3$, so all the three $v_{i}$ are vertices, and $\sum e\left(v_{i}\right)=1$. Since $\bar{e}=\sum e\left(v_{i}\right)-\sum\left|\gamma_{i}\right|$, by Theorem 2.1 we must have $\sum\left|\gamma_{i}\right|=1$, so there is one full edge in some $\gamma_{i}$. Because of symmetry $K$ is equivalent to $K(-1 / 3,-1 /(3+1 / n), 2 / 3)$. Since $\gamma_{3}$ is allowable, $n \neq-1$, 
and since $K$ is a knot, $n$ must be odd. This gives the knots listed in the lemma.

When $y_{2}=1 / 3$ or $-2 / 3, y_{3}=0$ or 1 , which is not a solution. When $y_{2}=2 / 3$ we have $y_{3}=-1 / 3$, which gives the same solution as above.

When $y_{2}=\frac{1}{4}$, we have $y_{3}=-y_{1}-y_{2}=\frac{1}{12}$. Now $v_{3}$ lies on the intersection of $y=\frac{1}{12}$ and $u=4$, which is a point on the edge $\langle 0,1 / 10\rangle$. We have

$$
0 \leq \overline{3} \leq \sum e\left(v_{i}\right)=\frac{1}{3}+0+\left(-\frac{2}{3}\right)<0
$$

hence it is not a solution.

When $y_{2}=-\frac{1}{4}, y_{3}=\frac{1}{3}+\frac{1}{4}=\frac{7}{12}$. One can check that the point of $(u, y)=\left(4, \frac{7}{12}\right)$ lies on the edge $\left\langle\frac{1}{2}, \frac{3}{5}\right\rangle$. We have $\beta_{3}=(\bar{u}-2) /(5-2)=1 / 3$. Since $\beta_{1}=\beta_{2}=0$, by Lemma 2.5 the boundary slope of the surface is $\delta \equiv$ $2\left(e_{-}-e_{+}\right) \equiv \pm 2 \beta_{3}= \pm 2 / 3 \bmod 1$, hence by Theorem 2.1 we have $\bar{e}=2 / 3$. On the other hand, we have

$$
\bar{e}=\sum e\left(\gamma_{i}\right)=\frac{1}{3}+0+\left(\frac{1}{3}(4-4)-\frac{1}{3}\right)=0 .
$$

This contradiction completes the proof of the lemma.

Lemma 3.6. Suppose $v_{1} \in L\left(-\frac{1}{2}\right), v_{2}$ is a vertex of $\mathcal{D}$, and $v_{3}$ is not in the interior of a horizontal edge. Then $K$ and $\bar{u}$ are given by one of the following.

(i) $K(-1 / 2,1 / 5,2 / 7), \bar{u}=5$;

(ii) $K(-1 / 2,2 / 5,1 / 9), \bar{u}=5$;

(iii) $K(-1 / 2,1 / 5,1 /(3+1 / n)), n$ even, $n \neq 0, \bar{u}=3$;

(iv) $K(-1 / 2,1 / 3,1 /(5+1 / n)), n$ even, $n \neq 0, \bar{u}=3$.

Proof. Let $v_{2}=\left\langle y_{2}\right\rangle=\left\langle p_{2} / q_{2}\right\rangle$. By definition of $e\left(\gamma_{i}\right)$ and Theorem 2.1 we have

$$
\begin{aligned}
0 \leq \bar{e}=\sum e\left(\gamma_{i}\right) & =\left(\frac{1}{3}+\frac{1}{6} \bar{u}\right)+2 \times \frac{1}{3}(4-\bar{u})-\left|\gamma_{2}\right|-\left|\gamma_{3}\right| \\
& =3-\frac{1}{2} \bar{u}-\left|\gamma_{2}\right|-\left|\gamma_{3}\right|
\end{aligned}
$$

which gives $\bar{u}=q_{2} \leq 6$. We have $y_{2}>0$ since otherwise $y_{3}=-y_{1}-y_{2}>$ $\frac{1}{2}$, so $\left|y_{1}\right|+\left|y_{3}\right|>1$, contradicting Lemma $2.4(2)$. Similarly we must have 
$y_{2} \leq \frac{1}{2}$ as otherwise we would have $\left|y_{1}\right|+\left|y_{2}\right|>1$. Moreover, $y_{2} \neq \frac{1}{2}$ as otherwise we would have $y_{3}=-y_{1}-y_{2}=0$, contradicting Lemma 2.4(3). Therefore $y_{2}=\frac{1}{3}, \frac{1}{4}, \frac{1}{5} \frac{2}{5}$, or $\frac{1}{6}$. In each case $v_{3}$ is uniquely determined by the facts that $u\left(v_{3}\right)=\bar{u}=q_{2}$, and $y_{3}=-y_{1}-y_{2}$. We separate the cases.

Case 1. $y_{2}=\frac{1}{3}$.

We have $\bar{u}=3$, and $y_{3}=\frac{1}{6}$. The point $v_{3}$ lies on the edge $\left\langle 0, \frac{1}{5}\right\rangle$, with $\beta_{3}=(5-3) /(5-1)=1 / 2$, which is the length of the last segment of $\gamma_{3}$. Hence

$$
\sum e\left(v_{i}\right)-\beta_{3}=\frac{5}{6}+\frac{1}{3}+\frac{1}{3}-\frac{1}{2}=1,
$$

so there is an extra edge, whose ending point is either $\langle 1 / 3\rangle$ or $\langle 1 / 5\rangle$. Since $K=K\left(-\frac{1}{2}, a_{2} / b_{2}, a_{3} / b_{3}\right)$ is a knot, the numbers $b_{2}$ and $b_{3}$ must be odd. Combining these, we see that $K$ is equivalent to a knot of type (iii) or (iv) in the Lemma.

Case 2. $y_{2}=\frac{1}{4}$.

We have $\bar{u}=4$ and $y_{3}=-y_{1}-y_{2}=\frac{1}{4}$, so $v_{3}$ is also at $\left\langle\frac{1}{4}\right\rangle$. Since $\sum e\left(v_{i}\right)=\left(\frac{1}{3}+\frac{1}{6} \bar{u}\right)+0+0=1$, there is one extra edge. It follows that $K=$ $K\left(-\frac{1}{2}, \frac{1}{4}, 1 /\left(4+\frac{1}{n}\right)\right)$, which is a link of at least two components. Therefore there is no solution in this case.

\section{Case 3. $y_{2}=\frac{1}{5}$.}

We have $\bar{u}=5$ and $y_{3}=\frac{1}{2}-\frac{1}{5}=\frac{3}{10}$. The vertex $v_{3}$ lies on the edge $\left\langle\frac{1}{3}, \frac{2}{7}\right\rangle$, and $\beta_{3}=(7-5) /(7-3)=1 / 2$. We have $\sum e\left(v_{i}\right)-\beta_{3}=\left(\frac{1}{3}+\frac{5}{6}\right)+$ $\frac{1}{3}(4-5)+\frac{1}{3}(4-5)-\frac{1}{2}=0$, so there is no extra edge. The knot is $K\left(-\frac{1}{2}\right.$, $\left.\frac{1}{5}, \frac{2}{7}\right)$.

Case 4. $y_{2}=\frac{2}{5}$.

Then $\bar{u}=5$ and $y_{3}=\frac{1}{10}$. The point $v_{3}$ lies on the edge $\left\langle 0, \frac{1}{9}\right\rangle$, and $\beta_{3}=$ $(9-5) /(9-1)=1 / 2$. We have $\sum e\left(v_{i}\right)-\beta_{3}=\left(\frac{1}{3}+\frac{5}{6}\right)+\frac{1}{3}(4-5)+\frac{1}{3}(4-$ $5)-\frac{1}{2}=0$, so there is no extra edge. The knot is $K\left(-\frac{1}{2}, \frac{2}{5}, \frac{1}{9}\right)$.

Case 5. $y_{2}=\frac{1}{6}$.

Then $\bar{u}=6$ and $y_{3}=\frac{1}{2}-\frac{1}{6}=\frac{1}{3}$. The point $v_{3}$ is in the interior of the horizontal line $L\left(\frac{1}{3}\right)$, which contradicts the assumption. Therefore there is no solution in this case.

Proposition 3.1. Suppose $v_{1} \in L$ and $\alpha_{i}=0$ for $i=2$ or 3 . Then $K$ is equivalent to one of the knots listed in Lemma 3.2, 3.5 or 3.6.

Proof. By symmetry we may assume that $\alpha_{2}=0$, so $v_{2}$ is either a vertex or in the interior of a horizontal line $L\left(p_{2} / q_{2}\right)$. The first case is covered by Lemmas 3.5 and 3.6. In the second case by Lemmas 3.3 and 3.4 we must have $q_{2} \leq 3$. Since $y_{2} \neq 0$, we have $q_{2} \geq 2$. We may now apply Lemma 3.2 unless 
$v_{1}$ is a vertex of $L$, which happens only if $q_{2}=2$ and $v_{1}=\left\langle-\frac{1}{3}\right\rangle$. If that is the case, then we may consider the (equivalent) knot $K\left(-p_{2} / q_{2},-p_{1} / q_{1},-p_{3} / q_{3}\right)$ instead, which has the property that the ending points of the corresponding edge paths are $\left(v_{1}^{\prime}, v_{2}^{\prime}, v_{3}^{\prime}\right)$, with $v_{1}^{\prime} \in L\left(-\frac{1}{2}\right)$ and $v_{2}^{\prime}=\left\langle\frac{1}{3}\right\rangle$, which has been covered by Lemma 3.6.

\section{The case that $v_{1} \in L\left(-\frac{1}{3}\right)$ and $\alpha_{i} \neq 0$ for $i=2,3$}

In this section we will assume that $v_{1} \in L\left(-\frac{1}{3}\right)$, and $v_{i}$ is in the interior of a non-horizontal edge $E_{i}=\left\langle p_{i} / q_{i}, r_{i} / s_{i}\right\rangle$ and hence $0<\alpha_{i}<1$ for $i=2,3$.

We have $\bar{u}=u\left(v_{1}\right) \geq 3$.

Define

$$
\begin{aligned}
\beta_{i}(u) & =\frac{s_{i}-u}{s_{i}-q_{i}}, \\
e(u) & =3-\frac{2}{3} u-\beta_{2}(u)-\beta_{3}(u) .
\end{aligned}
$$

Then $e(u)$ is a linear function of $u$. Recall that $\beta_{i}=\left(s_{i}-\bar{u}\right) /\left(s_{i}-q_{i}\right)=$ $\beta_{i}(\bar{u})$. We have

Lemma 4.1. Suppose $v_{1} \in L\left(-\frac{1}{3}\right)$, and $\alpha_{i} \neq 0$ for $i=2,3$. Then

(1) $0 \leq \bar{e} \leq e(\bar{u})$, and

(2) $3 \leq \bar{u}<4.5$.

Proof. (1) By definition we have

$$
\begin{aligned}
0 \leq \bar{e} & =\sum e\left(\gamma_{i}\right)=\frac{1}{3}+2 \times\left(\frac{1}{3}(4-\bar{u})\right)-\left|\gamma_{2}\right|-\left|\gamma_{3}\right| \\
& \leq 3-\frac{2}{3} \bar{u}-\beta_{2}-\beta_{3}=e(\bar{u}) .
\end{aligned}
$$

(2) Since $v_{i}$ is in the interior of non-horizontal edge, $\beta_{i}>0$ for $i=2,3$. Hence the above inequality implies that $\bar{u}<4.5$. Since $v_{1} \in L\left(-\frac{1}{3}\right)$, we have $\bar{u} \geq 3$.

Lemma 4.2. Suppose $v_{1} \in L\left(-\frac{1}{3}\right)$, and $\alpha_{i} \neq 0$ for $i=2,3$. Then $q_{i} \leq 3$ for $i=2,3$.

Proof. Since $q_{i} \leq \bar{u}$, by Lemma $4.1(2)$ we have $q_{i} \leq 4$ for $i=2,3$. If $q_{2}=4$ then by Lemma $4.1(1)$ we have $4<\bar{u}<4.5$, so $\beta_{2}=\left(s_{2}-\bar{u}\right) /\left(s_{2}-4\right) \geq 1 / 2$. 
Therefore $e(\bar{u})=3-\frac{2}{3} \bar{u}-\beta_{2}-\beta_{3}<0$, which is a contradiction to Lemma 4.1(1).

Lemma 4.3. Suppose $v_{1} \in L\left(-\frac{1}{3}\right)$ and $\alpha_{i} \neq 0$ for $i=2,3$. Then $q_{2} \leq 2$.

Proof. Assume to the contrary that $q_{2}>2$. Then by Lemma 4.2 we must have $q_{2}=3$. In this case $\beta_{2}(3)=-1$, so $e(3)=3-\frac{2}{3}(3)-1-\beta_{3}<0$.

First assume $s_{2} \geq 5$. If $s_{3} \geq 5$ then $\beta_{i}(5) \geq 0$ for $i=2,3$, so $e(5)=3-$ $\frac{2}{3} \times 5-\beta_{2}(5)-\beta_{3}(5)<0$, and by linearity we have $e(\bar{u})<0$, which is a contradiction. If $s_{3}=4$ then $3<\bar{u}<4$. Since $\beta_{2}(4) \geq \frac{1}{2}$ we have $e(4)<3-$ $\frac{2}{3} 4-\beta_{2}(4)<0$, which again contradicts the fact that $e(\bar{u}) \geq 0$.

We may now assume $s_{2}=4$. Then $3<\bar{u}<4$. We have $e(3)<0$, and $e(\bar{u}) \geq 0$, hence $e(4)=\frac{1}{3}-\beta_{3}(4)>0$, i.e., $\beta_{3}(4)=\left(s_{3}-4\right) /\left(s_{3}-q_{3}\right)<\frac{1}{3}$.

If $q_{3}=3$ then $\beta_{3}(4)<\frac{1}{3}$ implies that $s_{3}=4$. Since $\left|y_{i}\right| \leq \frac{2}{3}, E_{3} \neq\left\langle \pm \frac{2}{3}\right.$, $\left.\pm \frac{3}{4}\right\rangle$, so we must have $E_{3}=\left\langle \pm \frac{1}{3}, \pm \frac{1}{4}\right\rangle$. Since $\left(q_{2}, s_{2}\right)=(3,4)$, the same is true for $E_{2}$, hence $\frac{1}{4}<\left|y_{i}\right|<\frac{1}{3}$ for $i=2,3$. Therefore either $y_{2}+y_{3}=0$ (if $\left.E_{2} \neq E_{3}\right)$, or $\left|y_{2}+y_{3}\right|>\frac{1}{2}$, either case contradicting the fact that $y_{2}+y_{3}=$ $-y_{1}=\frac{1}{3}$.

If $q_{3}=2$ then since $s_{3}$ is coprime with $q_{3}, s_{3} \neq 4$, so we must have $s_{3} \geq 5$. Thus $\beta_{3}(4)=\left(s_{3}-4\right) /\left(s_{3}-2\right) \geq 1 / 3$, which is a contradiction.

If $q_{3}=1$ then $\beta_{3}(4)=\left(s_{3}-4\right) /\left(s_{3}-1\right)<1 / 3$ implies that $s_{3}=4$ or 5 , so $E_{3}=\left\langle 0, \pm \frac{1}{4}\right\rangle$ or $\left\langle 0, \pm \frac{1}{5}\right\rangle$. As above, $E_{2}=\left\langle \pm \frac{1}{3}, \pm \frac{1}{4}\right\rangle$, and one can check that there is no solution to the equation $y_{1}+y_{2}+y_{3}=0$ in these cases.

Proposition 4.1. Suppose $v_{1} \in L\left(-\frac{1}{3}\right)$, and $\alpha_{i} \neq 0$ for $i=2,3$. Then $K$ is equivalent to either $K(-1 / 3,1 / 5,1 / 5)$ or $K(-1 / 3,1 / 4,1 / 7)$, and $\bar{u}=3$.

Proof. First assume that $s_{2}, s_{3} \geq 5$. Then $\beta_{i}(5) \geq 0$, hence $e(5)=3-\frac{2}{3} \times$ $5-\beta_{2}(5)-\beta_{3}(5)<0$. By Lemma 4.1 we have

$$
e(3)=3-\frac{2}{3} \times 3-\beta_{2}(3)-\beta_{3}(3)=1-\beta_{2}(3)-\beta_{3}(3) .
$$

Since $e(\bar{u}) \geq 0$ for some $3 \leq \bar{u}<5$, by linearity we have $e(3) \geq 0$. Therefore, one of the $\beta_{i}$, say $\beta_{2}$, satisfies

$$
\beta_{2}(3)=\frac{s_{2}-3}{s_{2}-q_{2}} \leq \frac{1}{2}
$$

Since $s_{2} \geq 5$, this is true if and only if $\left(q_{2}, s_{2}\right)=(1,5)$, in which case $\beta_{2}(3)=\frac{1}{2}$, hence $\beta_{3}(3) \leq \frac{1}{2}$, and the same argument as above shows that 
$\left(q_{3}, s_{3}\right)=(1,5)$. It follows that $K=K(-1 / 3,1 / 5,1 / 5)$, and $\bar{u}=3$ because $e(\bar{u})<0$ if $\bar{u}>3$.

Now assume $s_{2}=4$. Then $3 \leq \bar{u}<4$. Since $q_{2}$ is coprime with $s_{2}$ and $q_{2}<3$ by Lemma 4.3 , we must have $q_{2}=1$, so $\left(q_{2}, s_{2}\right)=(1,4)$. Since $\left|y_{2}\right| \leq$ $\frac{2}{3}, E_{2} \neq\left\langle \pm 1, \pm \frac{3}{4}\right\rangle$. Therefore we must have $E_{2}=\left\langle 0, \pm \frac{1}{4}\right\rangle$.

Suppose $E_{2}=\left\langle 0,-\frac{1}{4}\right\rangle$. Then $-E_{1}-E_{2}$ coincides with the edge $\left\langle\frac{1}{2}, \frac{3}{5}\right\rangle$ on $3 \leq u \leq 4$, so $E_{3}=\left\langle\frac{1}{2}, \frac{3}{5}\right\rangle$ if there is a solution. By Example 2.1 and Definition 2.2 we have

$$
\bar{e}=\sum e\left(\gamma_{i}\right) \leq \sum e\left(v_{i}\right)-\beta_{3}=\frac{1}{3}+0+\frac{1}{3}(4-\bar{u})-\frac{5-\bar{u}}{5-2}=0 .
$$

On the other hand, since $E_{2}$ goes upward and $E_{3}$ downward when traveling from right to left, we have $e_{-} \equiv \beta_{3}$ and $e_{+} \equiv \beta_{2} \bmod 1$, hence by Lemma 2.5 the boundary slope of the surface $\delta$ satisfies

$$
\delta \equiv 2\left(e_{-}-e_{+}\right) \equiv 2\left(\beta_{3}-\beta_{2}\right)=2\left(\frac{5-\bar{u}}{5-2}-\frac{4-\bar{u}}{4-1}\right)=\frac{2}{3} .
$$

It follows from Theorem 2.1 that $\bar{e}=\frac{2}{3}$, which is a contradiction. Therefore, there is no solution in this case.

Now assume $E_{2}=\left\langle 0, \frac{1}{4}\right\rangle$. Then $y_{2}=x / 3$, and $y_{3}=-y_{1}-y_{2}=(1-x) / 3$. One can check that when $\bar{u}=3, v_{3}$ is on the edge $\left\langle 0, \frac{1}{7}\right\rangle$, in which case we have $\bar{e}=0$, and $K=K\left(-\frac{1}{3}, \frac{1}{4}, \frac{1}{7}\right)$. We need to show that there is no solution when $\bar{u}>3$.

The line segment $y=(1-x) / 3$ is below the line $y=1-x$, hence from figure 1 we see that $y_{3}$ is on an edge $E_{3}=\left\langle 0,1 / s_{3}\right\rangle$ for some $s_{3}$. Since the line segment has negative slope, and since it intersects $\left\langle 0, \frac{1}{7}\right\rangle$ at $u=3$, we must have $s_{3}>7$ when $u>3$. By definition we have

$$
\bar{e}=\sum e\left(\gamma_{i}\right)=\frac{1}{3}+0+\left(\frac{1}{3}(4-\bar{u})-\frac{s_{3}-\bar{u}}{s_{3}-1}\right) .
$$

For $s_{3}>7$, the right hand side is negative for $\bar{u}=3$ and 4 , hence by linearity it is negative for all $3 \leq \bar{u} \leq 4$. By Theorem 2.1 there is no solution in this case.

\section{The case that $v_{1} \in L\left(-\frac{1}{2}\right)$ and $\alpha_{i} \neq 0$, for $i=2,3$}

In this section, we will assume that $v_{1} \in L\left(-\frac{1}{2}\right)$, and $v_{i}$ is in the interior of a non-horizontal edge $E_{i}=\left\langle p_{i} / q_{i}, r_{i} / s_{i}\right\rangle$ and hence $0<\alpha_{i}<1$ for $i=2,3$. By 
Lemma 2.4 (2) and (3) we must have $0<y_{i}<\frac{1}{2}$ for $i=2,3$, hence $0 \leq z \leq \frac{1}{2}$ when $z=p_{i} / q_{i}$ or $r_{i} / s_{i}$ and $i=2,3$.

As before, define $\bar{e}=\sum e\left(\gamma_{i}\right)$, and $\beta_{i}(u)=\left(u-q_{i}\right) /\left(s_{i}-q_{i}\right)$. Let $\beta_{i}=$ $\beta_{i}(\bar{u})$. Define a function

$$
e(u)=3-\frac{1}{2} u-\beta_{2}(u)-\beta_{3}(u) .
$$

Note that this is different from the function $e(u)$ defined in Section 4.

Define $l_{i}=s_{i}-q_{i}$. Given an edge $E$ in $\mathcal{D}$ and a number $t$, we use $t-E$ to denote the set of points $\left\{t-t^{\prime} \mid t^{\prime} \in E\right\}$.

Lemma 5.1. Suppose $v_{1} \in L\left(-\frac{1}{2}\right)$ and $\alpha_{i} \neq 0$ for $i=2,3$. Then

$$
\begin{aligned}
0 \leq \bar{e} & \leq e(\bar{u})=3-\frac{1}{2} \bar{u}-\beta_{2}-\beta_{3}=1-\frac{1}{2} \bar{u}+\alpha_{2}+\alpha_{3} \\
& =1-\frac{1}{2} \bar{u}+\frac{\bar{u}-q_{2}}{l_{2}}+\frac{\bar{u}-q_{3}}{l_{3}} \\
& =\left(1-\frac{q_{2}}{l_{2}}-\frac{q_{3}}{l_{3}}\right)-\left(\frac{1}{2}-\frac{1}{l_{2}}-\frac{1}{l_{3}}\right) \bar{u}
\end{aligned}
$$

(2) $2 \leq \bar{u}<6$.

Proof. (1) By Theorem 2.1 we have $\bar{e}=\sum e\left(\gamma_{i}\right) \geq 0$. Since $v_{1} \in L\left(-\frac{1}{2}\right)$, by Definition 2.2 and Example 2.1 we have

$$
\bar{e}=\left(\frac{1}{3}+\frac{1}{6} \bar{u}\right)+2 \times \frac{1}{3}(4-\bar{u})-\left|\gamma_{2}\right|-\left|\gamma_{3}\right| \leq e(\bar{u})
$$

The other equalities are just different expressions of $e(\bar{u})$.

(2) Since $v_{1} \in L\left(-\frac{1}{2}\right)$, we have $\bar{u} \geq 2$. Since $v_{i}$ is in the interior of nonhorizontal edges, we have $\beta_{i}>0$, so $0 \leq e(\bar{u})<3-\frac{1}{2} \bar{u}$, hence $\bar{u}<6$.

Lemma 5.2. Suppose $v_{1} \in L\left(-\frac{1}{2}\right)$, and $\alpha_{i} \neq 0$ for $i=2,3$. Then $q_{i} \leq 3$ for $i=2,3$.

Proof. Note that $q_{i} \leq \bar{u}<6$. If $q_{2}=5$ then $\bar{u} \in[5,6)$. We have $e(6) \leq 3-\frac{1}{2} \times 6=0$, and $e(5)=3-\frac{5}{2}-\beta_{2}(5)-\beta_{3}(5)<0$ because $\beta_{2}(5)=$ 1. Since $e(u)$ is linear, $e(\bar{u})<0$, a contradiction. Therefore we may assume that $q_{2}=4$. 
First assume $s_{2}>5$. We have $e(4)=1-\beta_{2}(4)-\beta_{3}(4)=-\beta_{3}(4)<0$ and $e(\bar{u}) \geq 0$. If $s_{3}>5$ then $\beta_{i}(6) \geq 0$, so $e(6) \leq 3-\frac{1}{2} \times 6=0$, which contradicts $4<\bar{u}<6$ and the linearity of $e(u)$. If $s_{3}=5$ then $4<\bar{u}<5$, and $e(5)=3-\frac{1}{2} \times 5-\beta_{2}(5) \leq 0$, which again is a contradiction.

Now assume $s_{2}=5$. Then $E_{2}=\left\langle p_{2} / 4, r_{2} / 5\right\rangle$. Since $0<y_{2}<\frac{1}{2}$, we must have $E_{2}=\left\langle\frac{1}{4}, \frac{1}{5}\right\rangle$. However, one can check that the interior of the line segment $\frac{1}{2}-E_{2}$ lies in the interior of the triangle with vertices $\left\langle\frac{1}{3}\right\rangle,\left\langle\frac{1}{4}\right\rangle,\left\langle\frac{2}{7}\right\rangle$, hence there is no solution to the equation $\sum y_{i}=0$ for $v_{2} \in \operatorname{Int} E_{2}$. This contradiction completes the proof of the lemma.

Lemma 5.3. If $v_{1} \in L\left(-\frac{1}{2}\right), \alpha_{i}>0$ for $i=2,3$, and $q_{3} \leq q_{2}=3$, then $K=K(-1 / 2,2 / 5,1 / 7)$ and $\bar{u}=4$, or $K(-1 / 2,1 / 5,2 / 7)$ and $3<\bar{u}<5$.

Proof. Since $y_{2} \in\left(0, \frac{1}{2}\right)$, we must have $E_{2}=\left\langle 1 / 3, r_{2} / s_{2}\right\rangle$. From figure 1 we see that $r_{2} / s_{2} \geq \frac{1}{4}$, hence $y_{2}>\frac{1}{4}$, and $y_{3}=-y_{1}-y_{2}<\frac{1}{4}$. Since $q_{3} \leq 3$ and $y_{3}<1 / 4$, from figure 1 , we see that $p_{3} / q_{3}=0$, so $E_{3}=\left\langle 0,1 / s_{3}\right\rangle$ for some $s_{3} \geq 4$. As before, put $l_{i}=s_{i}-q_{i}$.

Since $y_{1}=-\frac{1}{2}$ and $y_{3}=-y_{1}-y_{2}=\frac{1}{2}-y_{2}$, we see that $v_{3}$ lies on the intersection of $E_{3}$ and $\frac{1}{2}-E_{2}$. When $E_{2}=\langle 1 / 3,1 / 4\rangle$, one can check that the interior of the line segment $1 / 2-E_{2}$ lies in the interior of the triangle $\langle 0,1 / 4,1 / 5\rangle$. Therefore there is no solution in this case.

When $E_{2}=\langle 1 / 3,2 / 5\rangle$, we have the following calculation.

$$
\begin{aligned}
y_{2} & =\frac{1}{3}+\frac{1}{2}\left(x-\frac{2}{3}\right)=\frac{x}{2} \\
y_{3} & =\frac{1}{l_{3}} x \quad \text { and } \\
y_{3} & =-y_{1}-y_{2}=\frac{1}{2}-y_{2}=\frac{1}{2}-\frac{x}{2}, \quad \text { hence } \\
x & =\frac{l_{3}}{l_{3}+2}, \\
\bar{u} & =\frac{1}{1-x}=\frac{l_{3}+2}{2} .
\end{aligned}
$$

Since $v_{2} \in\langle 1 / 3,2 / 5\rangle$ and is not a vertex, we have $3<\bar{u}<5$, so the above gives $4<l_{3}<8$. Since $K\left(-1 / 2,2 / 5,1 / s_{3}\right)$ is a knot, $s_{3}$ must be odd. Therefore the only possibility is that $s_{3}=7$, in which case $l_{3}=6, \bar{u}=4$, and the knot is $K(-1 / 2,2 / 5,1 / 7)$.

When $E_{2}=\langle 1 / 3,2 / 7\rangle, 1 / 2-E_{2}$ lies on the edges $\langle 0,1 / 5\rangle$ and $\langle 1 / 5,2 / 9\rangle$. Since $q_{3} \leq 3$, we must have $E_{3}=\langle 0,1 / 5\rangle$. Note that $l_{2}=l_{3}$ and the slopes of these edges add up to zero. Therefore by Lemma 2.5 all solution surfaces on 
these edges have the same boundary slope. We have $K=K(-1 / 2,1 / 5,2 / 7)$, and $3<\bar{u}<5$.

We have $s_{2} \neq 6$ because $s_{2}$ is coprime with $q_{2}$. Thus it remains to consider the case that $E_{2}=\left\langle 1 / 3, r_{2} / s_{2}\right\rangle$ for some $s_{2} \geq 8$. It is clear from figure 1 that $1 / 2-E_{2}$ does not intersect the interior of $\langle 0,1 / s\rangle$ for $s \leq 4$. Therefore, we may assume that $E_{3}=\left\langle 0,1 / s_{3}\right\rangle$ for some $s_{3} \geq 5$. We now have $l_{2}=s_{2}-q_{2} \geq 5$ and $l_{3}=s_{3}-q_{3} \geq 4$. Since $\bar{u}>q_{2}=3$, by Lemma 5.1 we have

$$
\begin{aligned}
\bar{e} & \leq\left(1-\frac{q_{2}}{l_{2}}-\frac{q_{3}}{l_{3}}\right)+\left(-\frac{1}{2}+\frac{1}{l_{2}}+\frac{1}{l_{3}}\right) \bar{u} \\
& =\left(1-\frac{3}{l_{2}}-\frac{1}{l_{3}}\right)+3\left(-\frac{1}{2}+\frac{1}{l_{2}}+\frac{1}{l_{3}}\right)+\left(-\frac{1}{2}+\frac{1}{l_{2}}+\frac{1}{l_{3}}\right)(\bar{u}-3) \\
& =\left(-\frac{1}{2}+\frac{2}{l_{3}}\right)+\left(-\frac{1}{2}+\frac{1}{l_{2}}+\frac{1}{l_{3}}\right)(\bar{u}-3) \\
& \leq\left(-\frac{1}{2}+\frac{2}{4}\right)+\left(-\frac{1}{2}+\frac{1}{5}+\frac{1}{4}\right)(\bar{u}-3)<0 .
\end{aligned}
$$

This contradicts Theorem 2.1. Therefore, there is no solution in this case.

Lemma 5.4. Suppose $v_{1} \in L\left(-\frac{1}{2}\right), \alpha_{i}>0$ for $i=2,3$, and $q_{3} \leq q_{2}=2$. Then $K=K\left(-\frac{1}{2}, \frac{1}{3}, \frac{1}{7}\right)$, and $\bar{u}=\frac{5}{2}$.

Proof. The minimum value of $y_{2}$ on edges of type $\left\langle 1 / 2, r_{2} / s_{2}\right\rangle$ is achieved at the vertex $\langle 1 / 3\rangle$ on $\left\langle\frac{1}{2}, \frac{1}{3}\right\rangle$. If $q_{3}=2$ then $y_{2}+y_{3} \geq 2 / 3>y_{1}$, so there is no solution to $\sum y_{i}=0$. Therefore we must have $q_{3}=1$. By the remark at the beginning of the section we have $y_{2}<1 / 2$, so $E_{2}=\left\langle 1 / 2, r_{2} / s_{2}\right\rangle$ for some $r_{2} / s_{2}<1 / 2$, and $E_{3}=\left\langle 0,1 / s_{3}\right\rangle$.

We have the following calculation.

$$
\begin{aligned}
& y_{2}=\frac{1}{2}-\frac{1}{l_{2}}\left(x-\frac{1}{2}\right) \\
& y_{3}=\frac{1}{l_{3}} x \text { and } \\
& y_{3}=\frac{1}{2}-y_{2}=\frac{1}{l_{2}}\left(x-\frac{1}{2}\right), \quad \text { hence } \\
& x=\frac{l_{3}}{2\left(l_{3}-l_{2}\right)}
\end{aligned}
$$




$$
\begin{aligned}
& \bar{u}=\frac{1}{1-x}=\frac{2\left(l_{3}-l_{2}\right)}{l_{3}-2 l_{2}}=2+\frac{2 l_{2}}{l_{3}-2 l_{2}}, \\
& \alpha_{2}=\frac{\bar{u}-q_{2}}{l_{2}}=\frac{\bar{u}-2}{l_{2}}=\frac{2}{l_{3}-2 l_{2}}, \\
& \alpha_{3}=\frac{\bar{u}-q_{3}}{l_{3}}=\frac{\bar{u}-1}{l_{3}}=\frac{1}{l_{3}-2 l_{2}}, \\
& 0 \leq \bar{e} \leq e(\bar{u})=1-\frac{1}{2} \bar{u}+\alpha_{2}+\alpha_{3}=\frac{3-l_{2}}{l_{3}-2 l_{2}} .
\end{aligned}
$$

Since $v_{2} \in\left\langle 1 / 2, r_{2} / s_{2}\right\rangle$ and is not a vertex, we have $\bar{u}<s_{2}=l_{2}+2$. By the above formula of $\bar{u}$, this gives $2+2 l_{2} /\left(l_{3}-2 l_{2}\right)<2+l_{2}$, hence

$$
l_{3}-2 l_{2}>2 \text {. }
$$

Note that the slope of $E_{2}$ is negative and the slope of $E_{3}$ is positive, so by Lemma 2.5 the boundary slope $\delta$ of the surface satisfies

$$
\delta \equiv 2\left(e_{-}-e_{+}\right) \equiv 2\left(\beta_{3}-\beta_{2}\right) \equiv 2\left(\alpha_{2}-\alpha_{3}\right)=\frac{2}{l_{3}-2 l_{2}} \not \equiv 0 \quad \bmod 1 .
$$

By Theorem 2.1 this means that $\frac{1}{2} \leq \bar{e}<1$, hence

$$
e(\bar{u})=\frac{3-l_{2}}{l_{3}-2 l_{2}}=\frac{1}{2} \times \frac{3-l_{2}}{\left(l_{3} / 2\right)-l_{2}} \geq \bar{e} \geq \frac{1}{2} .
$$

By $(*)$ we have $\left(l_{3} / 2\right)-l_{2}>0$, so the above inequality gives

$$
l_{3} \leq 6
$$

Together with $\left(^{*}\right)$, this implies that $\left(l_{2}, l_{3}\right)=(1,5)$ or $(1,6)$. By definition $e(\bar{u})-\bar{e}$ equals the number of full edges in $\cup e\left(\gamma_{i}\right)$. In both cases $e(\bar{u})<1$, so there are no full edges. In the first case we have $K=K(-1 / 2,1 / 3,1 / 6)$, which is not a knot. In the second case we have $\bar{u}=2.5, s_{2}=3$, and $s_{3}=7$, hence the knot is $K=K(-1 / 2,1 / 3,1 / 7)$. This solution gives the well-known $37 / 2$ toroidal surgery on $K(-1 / 2,1 / 3,1 / 7)$.

Lemma 5.5. Suppose $v_{1} \in L\left(-\frac{1}{2}\right), \alpha_{i}>0$, and $E_{i}=\left\langle 0,1 / s_{i}\right\rangle$ for $i=2,3$. Then

$$
\begin{array}{ll}
K=K(-1 /(2+1 / n), 1 / 3,1 / 3), & n \text { odd }, n \neq-1, \bar{u}=2 ; \text { or } \\
K=K(-1 / 2,1 / 3,1 /(3+1 / n)), & n \text { even, } n \neq 0, \bar{u}=2 .
\end{array}
$$


Proof. Since $v_{i}$ is in the interior of the edge $E_{i}$ above, we have $y_{i}<1 / s_{i}$, so $\sum y_{i}=0$ has no solution if $s_{i} \geq 4$ for $i=2,3$. Also since $v_{1} \in L\left(-\frac{1}{2}\right)$, we have $\bar{u} \geq 2$, hence $s_{i}>2$. Therefore we may assume that $s_{2}=3$ and $s_{3} \geq 3$. We have

$$
\begin{aligned}
& y_{2}+y_{3}=\frac{x}{l_{2}}+\frac{x}{l_{3}}=\left(\frac{1}{l_{2}}+\frac{1}{l_{3}}\right) x=\frac{1}{2}=-y_{1}, \\
& x=\frac{l_{2} l_{3}}{2\left(l_{2}+l_{3}\right)}, \\
& \bar{u}=\frac{1}{1-x}=\frac{2\left(l_{2}+l_{3}\right)}{2\left(l_{2}+l_{3}\right)-l_{2} l_{3}}, \\
& \alpha_{2}=\frac{\bar{u}-q_{2}}{l_{2}}=\frac{l_{3}}{2\left(l_{2}+l_{3}\right)-l_{2} l_{3}}, \\
& \alpha_{3}=\frac{l_{2}}{l_{3}}=\frac{q_{3}}{2\left(l_{2}+l_{3}\right)-l_{2} l_{3}}, \\
& \alpha_{2}+\alpha_{3}=\frac{1}{2} \bar{u}, \\
& \bar{e} \equiv e(\bar{u})=1-\frac{1}{2} \bar{u}+\alpha_{2}+\alpha_{3}=1 .
\end{aligned}
$$

Since both $E_{2}$ and $E_{3}$ have positive slope, $e_{-} \equiv \beta_{2}+\beta_{3} \equiv-\left(\alpha_{2}+\alpha_{3}\right)$ $\bmod 1$; by Lemmas 2.11 the boundary slope of the surface satisfies $\delta \equiv$ $2\left(e_{-}-e_{+}\right) \equiv-2\left(\alpha_{2}+\alpha_{3}\right)=-\bar{u}$. Since $\bar{e} \equiv 0 \bmod 1$, by Theorem 2.1 $\delta=-\bar{u}$ must be an integer slope, and $\bar{e}=0$. Since $v_{1} \in L\left(-\frac{1}{2}\right)$ and $v_{2}$ is in the interior of $\langle 0,1 / 3\rangle$, we have $2 \leq \bar{u}<3$, hence $\bar{u}=2$. From the formula of $\bar{u}$ above we have $l_{2}=l_{3}=2$. Also

$$
0=\bar{e}=e(\bar{u})-\sum\left(\left|\gamma_{i}\right|-\beta_{i}\right)=1-\sum\left(\left|\gamma_{i}\right|-\beta_{i}\right)
$$

Hence there is an extra edge, which may end at either $\left\langle\frac{1}{2}\right\rangle$ or $\left\langle\frac{1}{3}\right\rangle$. Therefore $K$ is one of the following knots.

$$
\begin{array}{ll}
K\left(-\frac{1}{2+1 / n}, \frac{1}{3}, \frac{1}{3}\right), & n \text { odd, and } n \neq-1 ; \\
K\left(-\frac{1}{2}, \frac{1}{3}, \frac{1}{3+1 / n}\right), & n \text { even, } n \neq 0 .
\end{array}
$$

The extra conditions on $n$ is to guarantee that $\gamma_{i}$ are allowable, and $K$ is a knot.

Proposition 5.1. If $v_{1} \in L\left(-\frac{1}{2}\right)$ and $\alpha_{i}>0$ for $i=2,3$, then $K$ is one of the knots in Lemma 5.3, 5.4 or 5.5 . 
Proof. Because of symmetry we may assume $q_{2} \geq q_{3} \geq 1$. By Lemma 5.2 we have $q_{2} \leq 3$, so $q_{2}=3,2$ or 1 , which are covered by Lemmas 5.3, 5.4 and 5.5 , respectively.

\section{No horizontal edges}

In this section, we study the case that no $v_{i}$ is on a horizontal edge. As before, let $E_{i}=\left\langle p_{i} / q_{i}, r_{i} / s_{i}\right\rangle$. By Lemma 2.4(4) we may assume that $v_{1}$ is in the interior of a non-horizontal edge in the graph $G$ shown in figure 4, hence $1<\bar{u}<3$ and $\bar{u} \neq 2$. Since $q_{i} \leq \bar{u}$, we have $q_{i} \leq 2$ for all $i$.

Similar to the previous sections, we define

$$
\begin{aligned}
\beta_{i}(u) & =\frac{s_{i}-u}{s_{i}-q_{i}}, \\
\alpha_{i}(u) & =\frac{u-q_{i}}{s_{i}-q_{i}} \\
e(u) & =4-u-\sum \beta_{i}(u)=1-u+\sum \alpha_{i}(u) .
\end{aligned}
$$

Since no $v_{i}$ is in the interior of a horizontal edge, by Definition 2.2 we have

$$
\begin{aligned}
\bar{e} & =\sum e\left(\gamma_{i}\right)=(4-\bar{u})-\sum\left|\gamma_{i}\right| \\
& \leq 4-\bar{u}-\sum \beta_{i}=1-\bar{u}+\sum \alpha_{i}=e(\bar{u}) .
\end{aligned}
$$

Note also that $e(\bar{u})-\bar{e}$ is a nonnegative integer, which equals the number of full edges in $\cup \gamma_{i}$.

Let $\delta$ be the boundary slope of $F\left(\gamma_{1}, \gamma_{2}, \gamma_{3}\right)$. Then

$$
\delta \equiv 2\left(e_{-}-e_{+}\right) \equiv-2 \sum \operatorname{sign}\left(r_{i} / s_{i}-p_{i} / q_{i}\right) \alpha_{i} \bmod 1 .
$$

Lemma 6.1. Suppose $v_{1} \in G-L$. If $q_{i}=2$, then $s_{i}=3$.

Proof. Assume to the contrary that $q_{2}=2$ and $s_{2}>3$. We have $E_{2}=\langle \pm 1 / 2$, $\left.r_{2} / s_{2}\right\rangle$. From figure 1 we see that any point $\left(x, y_{2}\right)$ in the interior of $E_{2}$ satisfies $x / 2<\left|y_{2}\right|<x$.

First assume that $s_{3}=3$. Denote by $\pm Q=\left\langle 0, \pm \frac{1}{3}\right\rangle \cup\left\langle \pm \frac{1}{2}, \pm \frac{1}{3}\right\rangle \cup\left\langle \pm \frac{1}{2}\right.$, $\left.\pm \frac{2}{3}\right\rangle$. Note that any point $(x, y)$ on $\pm Q$ satisfies $x / 2 \leq|y| \leq x$.

Since $\bar{u}>q_{2}=2$, we have $s_{1}=3$, so $v_{1} \in G-L$ implies that $v_{1} \in-Q$. We assumed $s_{3}=3$ and by Lemma 2.4 we have $\left|y_{3}\right| \leq \frac{2}{3}$, hence $v_{3} \in \pm Q$. Thus $x / 2 \leq\left|y_{i}\right| \leq x$ for $i=1,3$. This implies that if $y_{3}<0$ then $-y_{1}-y_{3} \geq$ $(x / 2)+(x / 2)=x$, and if $y_{3}<0$ then $\left|-y_{1}-y_{3}\right| \leq x-x / 2=x / 2$, either case contradicting the fact that $y_{2}=-y_{1}-y_{3}$ satisfies $x / 2<\left|y_{2}\right|<x$. 
We may now assume that $s_{3}>3$. Consider the function $e(u)=1-u+$ $\sum \alpha_{i}(u)$, where $\alpha_{i}(u)=\left(u-q_{i}\right) /\left(s_{i}-q_{i}\right)$. By (6.1) we have $e(\bar{u}) \geq \bar{e} \geq 0$.

When $u=2$ we have $\alpha_{1}(2) \leq \frac{1}{2}, \alpha_{2}(2)=0$ because $q_{2}=2$, and $\alpha_{3}(2) \leq \frac{1}{3}$ because $s_{3} \geq 4$, hence $e(2)=(1-2)+\sum \alpha_{i}(u)<0$.

Now calculate $e(3)$. Since $s_{2}$ is coprime with $q_{2}=2$ and $s_{2}>3$, we have $s_{2} \geq 5$. Hence $\alpha_{2}(3) \leq \frac{1}{3}$. Also, $\alpha_{3}(3) \leq \frac{2}{3}$ because $s_{3}>3$, and $\alpha_{1}(3) \leq 1$. Hence $e(3)=(1-3)+\sum \alpha_{i}(3) \leq 0$. By the linearity of $e(u)$ we have $\bar{e} \leq$ $e(\bar{u})<0$ because $2=q_{2}<\bar{u}<s_{1}=3$. This contradicts Theorem 2.1.

Lemma 6.2. Suppose $v_{1} \in \operatorname{Int}\left\langle-\frac{1}{2},-\frac{2}{3}\right\rangle$, and $\left|y_{1}\right|>y_{2} \geq y_{3}>0$. Then $\bar{u}=2.5$, and $K=K(-2 / 3,1 / 3,1 / 4)$.

Proof. The equation for $E_{1}$ is $y_{1}=-x$. By Lemma 2.4(2) we have $\left|y_{1}\right|+$ $\left|y_{2}\right| \leq 1$, hence $y_{2} \leq 1-x$. Since $y_{2}+y_{3}=-y_{1}$ and $y_{2} \geq y_{3}$, we have $y_{2} \geq$ $\frac{1}{2}\left(-y_{1}\right)=\frac{1}{2} x$. From figure 1 we see that $E_{2}=\left\langle\frac{1}{2}, \frac{1}{3}\right\rangle$ or $\left\langle 0, \frac{1}{3}\right\rangle$.

If $E_{2}=\left\langle 0, \frac{1}{3}\right\rangle$ then $y_{3}=-y_{1}-y_{2}=x-x / 2=x / 2=y_{2}$, so $E_{3}=E_{2}$. In this case $E_{1}=-E_{2}-E_{3}$, so there are infinitely many solutions, all giving the same slope. We have $\alpha_{1}=\bar{u}-2, \alpha_{2}=\alpha_{3}=(\bar{u}-1) / 2$, hence

$$
\bar{e} \leq e(\bar{u})=1-\bar{u}+\sum \alpha_{i}=\bar{u}-2<1 .
$$

Therefore there are no extra edges, hence $K=K\left(-\frac{2}{3}, \frac{1}{3}, \frac{1}{3}\right)$. Since this is a link of two components. there is no solution in this case.

Now assume $E_{2}=\left\langle\frac{1}{2}, \frac{1}{3}\right\rangle$. Then $y_{3}=-y_{1}-y_{2}=x-(1-x)=2 x-1<$ $\frac{1}{3}$ because $x<\frac{2}{3}$. Hence from figure 1 we see that $E_{3}=\left\langle 0,1 / s_{3}\right\rangle$ for some $s_{3} \geq 3$. Define $l_{i}=s_{i}-q_{i}$. We have the following calculation.

$$
\begin{aligned}
& -y_{1}-y_{2}=2 x-1=\frac{x}{l_{3}}=y_{3}, \\
& x=\frac{1}{2-1 / l_{3}}=\frac{l_{3}}{2 l_{3}-1}, \\
& \bar{u}=\frac{1}{1-x}=2+\frac{1}{l_{3}-1}, \\
& \alpha_{1}=\frac{\bar{u}-2}{l_{1}}=\frac{1}{l_{3}-1}, \\
& \alpha_{2}=\frac{\bar{u}-2}{l_{2}}=\frac{1}{l_{3}-1}, \\
& \alpha_{3}=\frac{\bar{u}-1}{l_{3}}=\frac{1}{l_{3}-1}, \\
& 0 \leq \bar{e} \leq e(\bar{u})=1-\bar{u}+\sum \alpha_{i}=-1+\frac{2}{l_{3}-1} .
\end{aligned}
$$


This gives $l_{3}=2$ or 3 . When $l_{3}=2, \bar{u}=3$, which is a contradiction because $\bar{u}<3$. When $l_{3}=3$, we have $\bar{u}=2.5$ and $\bar{e}=0$, so there is no extra edge, hence $K=K\left(r_{1} / s_{2}, r_{2} / s_{2}, r_{3} / s_{3}\right)=K(-2 / 3,1 / 3,1 / 4)$.

Lemma 6.3. Suppose $v_{1} \in \operatorname{Int}\langle-1 / 2,-1 / 3\rangle$, and $\left|y_{1}\right|>y_{2} \geq y_{3}>0$. Then $K=K(-1 / 3,1 / 3,1 / 7)$ and $\bar{u}=2.5$.

Proof. Since $-y_{1}>y_{2} \geq y_{3}>0, E_{2}$ and $E_{3}$ must be below the edge $E_{1}=$ $\left\langle\frac{1}{2}, \frac{1}{3}\right\rangle$, hence we must have $E_{i}=\left\langle 0,1 / s_{i}\right\rangle$ for $i=2,3$. We have the following calculation.

$$
\begin{aligned}
& \sum y_{i}=(x-1)+x / l_{2}+x / l_{3}=0, \\
& x=\frac{1}{\left(1 / l_{2}\right)+\left(1 / l_{3}\right)+1}, \\
& \bar{u}=\frac{1}{1-x}=1+\frac{l_{2} l_{3}}{l_{2}+l_{3}}, \\
& \alpha_{1}=\frac{\bar{u}-2}{l_{1}}=\bar{u}-2=\frac{l_{2} l_{3}}{l_{2}+l_{3}}-1, \\
& \alpha_{2}=\frac{\bar{u}-1}{l_{2}}=\frac{l_{3}}{l_{2}+l_{3}}, \\
& \alpha_{3}=\frac{\bar{u}-1}{l_{3}}=\frac{l_{2}}{l_{2}+l_{3}}, \\
& \delta \equiv 2\left(-\alpha_{1}-\alpha_{2}-\alpha_{3}\right) \equiv \frac{-2 l_{2} l_{3}}{l_{2}+l_{3}} \equiv-2 \bar{u}, \\
& 0 \leq \bar{e} \leq 1-\bar{u}+\sum \alpha_{i}=0 .
\end{aligned}
$$

The last inequality gives $\bar{e}=0$, so by Theorem 2.1 the boundary slope of the surface must be an integer, hence by Lemma 2.5 we have $\delta \equiv 2 \bar{u} \equiv 0 \bmod 1$. Since $2<\bar{u}<3$, we have $\bar{u}=2.5$. The only solutions for $\bar{u}=1+l_{2} l_{3} /\left(l_{2}+\right.$ $\left.l_{3}\right)=2.5$ are $\left(l_{2}, l_{3}\right)=(2,6)$ or $(3,3)$. Since $e=0$, there is no extra edge, so in the second case we would have $K=K(-1 / 3,1 / 4,1 / 4)$, which is not a knot. Therefore the only solution in this case is $K=K(-1 / 3,1 / 3,1 / 7)$ and $\bar{u}=5 / 2$.

Lemma 6.4. Suppose $v_{1} \in G$, and $\left|y_{1}\right|>y_{2} \geq y_{3}>0$. Then $v_{1} \notin \operatorname{Int}\langle 0$, $-1 / 2\rangle$.

Proof. If $v_{1} \in \operatorname{Int}\langle 0,-1 / 2\rangle$, then $\left|y_{1}\right|>y_{i}>0$ implies that $E_{i}=\left\langle 0,1 / s_{i}\right\rangle$ for some $s_{i} \geq 3$. We have $y_{1}=-x$, and $y_{i}=x / l_{i}$ for $i=2,3$, hence from the 
equations $y_{1}+y_{2}+y_{3}=0$ and $x>0$ we have

$$
-1+\frac{1}{l_{2}}+\frac{1}{l_{3}}=0
$$

Since $s_{i} \geq 3$, this gives $l_{2}=l_{3}=2$. Note that $\alpha_{1}=\bar{u}-1$, and $\alpha_{2}=\alpha_{3}=$ $(\bar{u}-1) / 2<1 / 2$, hence

$$
\bar{e}=4-\bar{u}-\sum\left|\gamma_{i}\right| \equiv 1-\bar{u}+\sum \alpha_{i}=\alpha_{2}+\alpha_{3}=\bar{u}-1 \not \equiv 0 \quad \bmod 1 .
$$

By Theorem 2.1 this implies that the boundary slope $\delta$ of the surface is not an integer slope. On the other hand, since $E_{1}$ has positive slope and $E_{2}, E_{3}$ have negative slope, by Lemma 2.5 we have

$$
\delta \equiv 2\left(e_{-}-e_{+}\right)=2\left(\beta_{2}+\beta_{3}-\beta_{1}\right) \equiv 2\left(-\alpha_{2}-\alpha_{3}+\alpha_{1}\right)=0 \quad \bmod 1
$$

so $\delta$ is an integer slope, which is a contradiction.

Lemma 6.5. Suppose $v_{1} \in \operatorname{Int}\langle 0,-1 / 3\rangle$, and $\left|y_{1}\right|>y_{2} \geq y_{3}>0$. Then $K=$ $K(-1 / 3,1 / 4,1 / 7)$ or $K(-1 / 3,1 / 5,1 / 5)$, and the boundary slopes are the same as the pretzel slopes corresponding to the candidate systems in Proposition 2.2.

Proof. Similar to the proof of Lemma 6.4, we have

$$
\sum y_{i}=-\frac{1}{2}+\frac{1}{l_{2}}+\frac{1}{l_{3}}=0
$$

which gives $\left(l_{2}, l_{3}\right)=(3,6)$ or $(4,4)$. We have

$$
0 \leq \bar{e} \leq e(\bar{u})=1-\bar{u}+\sum \alpha_{i}(\bar{u})=1-\bar{u}+\frac{\bar{u}-1}{2}+\frac{\bar{u}-1}{l_{2}}+\frac{\bar{u}-1}{l_{3}}=0 .
$$

Therefore there are no extra edges. The knots are $K=K(-1 / 3,1 / 4,1 / 7)$ and $K(-1 / 3,1 / 5,1 / 5)$. The boundary slopes are the same for all $\bar{u}$, which is also the boundary slope of the candidate system at $\bar{u}=1$, as given in Proposition 2.2.

Lemma 6.6. Suppose $v_{1} \in G$, and $\left|y_{1}\right|>y_{2} \geq y_{3}>0$. Then $v_{1} \notin \operatorname{Int}\langle-1$, $\left.-\frac{1}{2}\right\rangle$.

Proof. If $v_{1} \in \operatorname{Int}\left\langle-1,-\frac{1}{2}\right\rangle$ then $1<\bar{u}<2$, and we have $E_{i}=\left\langle 0,1 / s_{i}\right\rangle$ for $i=2,3$. If both $s_{i}>2$ then it is easy to show that $\left|y_{1}\right|>\left|y_{2}\right|+\left|y_{3}\right|$ for 
$0<x<\frac{1}{2}$, so there is no solution to $\sum y_{i}=0$ when $1<\bar{u}<2$. Hence we must have $s_{2}=2$, so $y_{1}=-1+x, y_{2}=x$, and $y_{3}=x / l_{3}$. We have the following calculations.

$$
\begin{aligned}
& \sum y_{i}=(-1+x)+x+\frac{x}{l_{3}}=0, \\
& x=\frac{l_{3}}{2 l_{3}+1}, \\
& \bar{u}=\frac{1}{1-x}=2-\frac{1}{l_{3}+1}=2-\frac{1}{s_{3}}, \\
& \alpha_{1}=\frac{\bar{u}-q_{1}}{l_{1}}=\frac{\left(2-1 / s_{3}\right)-1}{1}=1-\frac{1}{s_{3}}, \\
& \alpha_{2}=\frac{\bar{u}-q_{2}}{l_{2}}=\frac{\left(2-1 / s_{3}\right)-1}{1}=1-\frac{1}{s_{3}}, \\
& \alpha_{3}=\frac{\bar{u}-q_{3}}{l_{3}}=\frac{\left(2-1 / s_{3}\right)-1}{s_{3}-1}=\frac{1}{s_{3}}, \\
& \delta \equiv 2\left(-\alpha_{1}-\alpha_{2}-\alpha_{3}\right)=\frac{2}{s_{3}}, \\
& \bar{e} \equiv e(\bar{u})=1-\bar{u}+\sum \alpha_{i}=1 \equiv 0 \bmod 1 .
\end{aligned}
$$

By Theorem 2.1, $\bar{e} \equiv 0 \bmod 1$ implies that $\delta$ is an integer slope, hence from $\delta \equiv 2 / s_{3} \bmod 1$ and $s_{3} \geq 2$ we see that $s_{3}=2$. Since $\bar{e}=1$ there is one extra edge, but since $\left(r_{1} / s_{1}, r_{2} / s_{2}, r_{3} / s_{3}\right)=(-1 / 2,1 / 2,1 / 2)$ or $(-1 / 2,1 / 2,1 / 4)$, adding one extra edge will make a link of type $K(-1 / 2,1 / 2,1 /(2+1 / n))$ or $K(-1 / 2,1 / 2,1 /(4+1 / n))$, which has at least two components. Therefore there is no solution in this case.

Proposition 6.1. Suppose some $v_{i} \in G-L$, and $\bar{u}>1$. Then $K$ and $\bar{u}$ are equivalent to one of the pairs in Lemmas 6.2, 6.3 and 6.5.

Proof. Since $\bar{u}>1$, we must have $y_{j} \neq 0$. Up to permutation and changing of signs of the parameters of $K$ we may assume that $-y_{1} \geq\left|y_{i}\right|$ for $i=2,3$. Before this modification we have some $v_{i} \in G-L$. We need to show that $v_{1} \in G-L$ after the modification.

The assumption of $v_{i} \in G-L$ implies that $\bar{u}<3$, hence $p_{1} / q_{1} \in\left\{0,-\frac{1}{2}\right.$, $-1\}$. By Lemma 2.4(2) we have $\left|y_{1}\right| \leq \frac{2}{3}$, so from figure 1 we see that if $p_{1} / q_{1}=-1$ then $E_{1}=\left\langle-1,-\frac{1}{2}\right\rangle$, hence $v_{1} \in G-L$. If $p_{1} / q_{1}=-\frac{1}{2}$ then by Lemma 6.1 we have $r_{1} / s_{1}=-\frac{1}{3}$ or $-\frac{2}{3}$, so again $v_{1} \in G-L$. If $p_{1} / q_{1}=0$ and $v_{1} \notin G-L$ then from figure 1 and the assumption of $\left|y_{i}\right| \leq\left|y_{1}\right|$ we see that each $v_{j}$ is in $\left\langle 0, \pm \frac{1}{s_{j}}\right\rangle$ for some $s_{j} \geq 4$, which contradicts the assumption that some $v_{i}$ is in $G-L$ before the modification of parameters of $K$. 
We now have $v_{1} \in G-L$, and $-y_{1} \geq\left|y_{i}\right|$ for $i=2$, 3. Since $\bar{u}>1$, no $v_{i}$ is on the horizontal line $L(0)$ as otherwise the corresponding parameter of $K$ would be 0 , contradicting the assumption that the length of $K$ is 3. Hence from $\sum y_{i}=0$ we have that $\left|y_{1}\right|>y_{i}>0$. Permuting the second and third parameters of $K$ if necessary we may assume $y_{2} \geq y_{3}$. Therefore we have $\left|y_{1}\right|>y_{2} \geq y_{3}>0$.

There are only 5 edges in $G-L$, so $E_{1}$ must be one of them, which have been discussed in Lemma 6.2-6.6 respectively. The above discussion shows that $y_{i}$ satisfy the conditions of the lemmas, hence the result follows from these lemmas.

\section{The classification}

Lemma 7.1. Let $K$ be a Montesinos knot of length 3 . Then $E(K)$ contains a candidate surface $F$ of genus one with boundary slope $\delta$ if and only if $(K, \delta)$ is equivalent to one of the pairs listed in Theorem 1.1.

Proof. When $\bar{u} \leq 1$, the knots are given in Proposition 2.2. When $\bar{u}>1$, by Lemma 2.4(4) we may assume that $v_{1} \in G$. Propositions 3.7, 4.4 and 5.6 covered the case of $v_{1} \in L$, and Proposition 6.1 covered the case of $v_{1} \in G-$ $L$. The list in Theorem 1.1 contains all the knots given in these propositions. Here are more details.

Parts (1) and (2) of Theorem 1.1 include the knots in Proposition 2.2, as well as $K(-1 / 3,1 / 5,1 / 5)$ and $K(-1 / 3,1 / 4,1 / 7)$ in Proposition 4.1 and Lemma 6.5. Note that the boundary slopes for the last two are the same as those in the list, but the $\bar{u}$ values are different, which is allowed by the remark before the statement of Theorem 1.1.

Part (3) is given by Lemma 3.2, and part (4) by Lemma 3.5. Parts (5), (6) and (9) are in Lemma 3.6. Parts (7) and (8) are from Lemma 5.5, Parts (10) from Lemma 5.3, Parts (11) from Lemma 5.4, Parts (12) from Lemma 6.2, and (13) from Lemma 6.3. Note that the knot $K(-1 / 2,1 / 5,2 / 7)$ in Lemmas 3.6 and 5.3 is included in Parts (5) (with $n=2$ ) because they all have the same boundary slope. The boundary slopes can be calculated using the algorithm of Hatcher and Oertel in Lemma 2.5.

Proof of Theorem 1.1. Most of the work has been done throughout the paper and summarized in Lemma 7.1, as it was shown that the list in Theorem 1.1 contains all possible $(K, \delta)$. It remains to show that the candidate surfaces in Lemma 7.1 are incompressible in $E(K)$ when $K$ is hyperbolic. Since the candidate systems are already determined in the proofs of the lemmas and 
the propositions above, it is straightforward to follow the procedure of [4] to verify the incompressibility of the candidate surface $F$. For each individual knot the toroidal slopes can also be verified using the computer program of Nathan Dunfield [16].

Here are more details. If $\bar{u} \geq q_{i}$ for some $i$ then the path $\gamma_{i}$ is a constant path, so it follows from [4, Proposition 2.1] that $F$ is incompressible. This takes care of all the cases except (1), (2), (7), (12) and (13) in Theorem 1.1. Recall from [4, Page 463] that the $r$-value of a path is determined by this rule: Extend the last segment of the path to meet the right-hand border of the the diagram in a point whose slope has denominator $r$. (For length 3 knot we do not have vertical line segments in $\gamma_{i}$ and hence $r \neq 0$.) In this case by [4, Corollary 2.4] $F$ is incompressible unless the $r$-value cycle is $\left(1,1, r_{3}\right)$ or $\left(1,2, r_{3}\right)$. In cases (1) and (2) the $r$-value cycle is $\left(\left|q_{1}\right|-1,\left|q_{2}\right|-1,\left|q_{3}\right|-1\right)$, so $F$ is incompressible unless $\left|q_{1}\right|=2$ and $\left|q_{2}\right|=3$. $\left(\left|q_{2}\right| \neq 2\right.$ since $K$ is a knot.) By [4, Proposition 2.7] $F$ is incompressible unless the slopes of $\gamma_{1}$ is of opposite sign to those of $\gamma_{2}$ and $\gamma_{3}$ and $r_{3}=\left|q_{3}\right|-1=2$ or 4 . It follows that $F$ is incompressible unless $K$ is the non-hyperbolic knot $K(-1 / 2,1 / 3,1 / 3)$ or $K(-1 / 2,1 / 3,1 / 5)$, which has been excluded since we assumed that $K$ is hyperbolic. In case (7) $r_{i} \neq 1$ unless $K=K(-1 / 3,1 / 3,1 / 3)$, in which case the $r$-cycle is $(1,2,2)$. Since the slopes of the three edges are all positive (the first one is on $\langle-1 / 2,-1 / 3\rangle$ and the other two are on $(\langle 0 / 1,, 1 / 3\rangle)$, by [4, Proposition 2.7] $F$ is incompressible. In case (12) the $r$-cycle is $(1,1,3)$ and the first two paths are edges of the same slope - 1 , so $F$ is incompressible by [4, Proposition 2.6]. In case (13) the $r$-cycle is $(1,2,6)$ and the edges all have positive slopes, hence again the incompressibility of $F$ follows from [4, Proposition 2.7].

If $F$ is a surface in a three-manifold $M$, denote by $M \mid F$ the manifold obtained by cutting $M$ along $F$. Similarly, if $C$ is a set of curves on a surface $F$ then $F \mid C$ denotes the surface obtained by cutting $F$ along $C$. All surfaces in three-manifolds below are assumed compact, connected, orientable, and properly embedded. Recall that a surface in $M$ is essential if it is incompressible, $\partial$-incompressible, and is not boundary parallel. Denote by $|\partial F|$ the number of boundary components of $F$.

Lemma 7.2. Let $M$ be a compact orientable 3-manifold with $\partial M=T a$ torus. Let $F$ be a genus one separating essential surface in $M$ such that $|\partial F| \leq 4$. Let $M_{1}, M_{2}$ be the components of $M \mid F$, and let $A$ be a component of $\partial M_{1}-F$. If $F \cup A$ is incompressible in $M_{1}$, then $M$ contains a closed essential surface. 
Proof. This is due to Gordon, and is true for any number of components on $\partial F$. If $|\partial F|=2$ then by assumption $F \cup A$ is incompressible and the result follows, so we assume $|\partial F|=4$. Let $A^{\prime}$ be the annulus on $T$ which contains $A$ and such that $\partial A^{\prime}=\partial F-\partial A$. Then we can push the part of $F \cup A$ near $A$ into the interior of $M$ to obtain a surface $F^{\prime}$ with $\partial F^{\prime}=A^{\prime}$, and then push $F^{\prime} \cup A^{\prime}$ into the interior of $M$ to obtain a closed surface $F^{\prime \prime}$. Number the annuli $T \mid \partial F$ successively as $A_{1}, \ldots, A_{4}$, with $A=A_{1}$. One can show that $M \mid F^{\prime \prime}$ consists of two components $M_{1}^{\prime \prime}, M_{2}^{\prime \prime}$, such that $M_{1}^{\prime \prime}$ is obtained by gluing $T \times I$ to $M_{1}$ along the annulus $A_{3}$ and a non-trivial annulus on $T \times 0$, and $M_{2}^{\prime \prime}$ is obtained by gluing a $A^{\prime} \times I$ to $M_{2}$, where $A^{\prime}$ is an annulus, $A^{\prime} \times 0$ identified to $A_{2}$, and $A^{\prime} \times 1$ to $A_{4}$. An innermost circle argument shows that if $F \cup A$ is incompressible then $F^{\prime \prime}$ is incompressible in both $M_{i}^{\prime \prime}$, hence is incompressible in $M$.

Lemma 7.3. Let $M$ be a compact orientable irreducible three-manifold with $\partial M=T$ a torus. Let $F$ be a genus one separating incompressible surface in $M$ with boundary slope $\delta$, and let $\hat{F}$ be the corresponding torus in the Dehn filling manifold $M(\delta)$. If (i) $M$ contains no closed incompressible surface, and (ii) $F$ has at most four boundary components, then $\hat{F}$ is incompressible in $M(\delta)$.

Proof. Since $F$ is separating, $|\partial F|=2$ or 4 . We assume the latter, as the proof for the former case is similar and simpler. Let $M_{1}, M_{2}$ be the components of $M \mid F$, and let $A_{1}, \ldots, A_{4}$ be the annuli $T \mid \partial F$, labeled so that $\partial M_{1}=F \cup A_{1} \cup A_{3}$.

Since $M$ contains no closed essential surface, each $M_{i}$ is a handlebody of genus 3, and by Lemma 7.2 the surface $F_{1}=F \cup A_{1}$ is compressible in $M_{1}$. Let $D$ be a compressing disk of $F_{1}$. If $D$ is separating then since $M_{1}$ is a handlebody and $\partial M_{1}-F_{1}=A_{3}$ is connected, we can find a nonseparating compressing disk in a component of $M_{1} \mid D$ disjoint from $A_{3}$. Therefore, we may assume without loss of generality that $D$ is a nonseparating compressing disk. It follows that after attaching a two-handle to $M_{1}$ along $A_{3}$ the resulting manifold $M^{\prime}$ has compressible boundary, because $D$ remains a compressing disk of $\partial M^{\prime}$ in $M^{\prime}$.

We have $A_{1} \subset \partial M^{\prime}$. We want to show that $F_{2}=\partial M^{\prime}-A_{1}$ is incompressible in $M^{\prime}$. Consider the surface $F_{3}=F \cup A_{3}=\partial M_{1}-A_{1}$. For the same reason as above, we know that $F_{3}$ is compressible in $M_{1}$. By assumption $F_{3}-A_{3}=F$ is incompressible. Therefore, by the Handle Addition Lemma (see [17] or [18]), we know that after attaching a two-handle to $M_{1}$ along $A_{3}$, the resulting surface $F_{2}=\partial M^{\prime}-A_{1}$ is incompressible in $M^{\prime}$. 
We have shown that $\partial M^{\prime}$ is compressible, and $\partial M^{\prime}-A_{1}=F_{2}$ is incompressible. Applying the Handle Addition Lemma again, we see that after attaching a two-handle to $A_{1}$ the boundary of the resulting manifold $M^{\prime \prime}$ is incompressible. Note that $M^{\prime \prime}$ is a component of $M(\delta) \mid \hat{F}$, and $\partial M^{\prime \prime}=\hat{F}$. For the same reason, $\hat{F}$ is incompressible in the other component of $M(\delta) \mid \hat{F}$. It follows that $\hat{F}$ is incompressible in $M(\delta)$.

Remark. The above result is similar to a special case of Proposition 2.2.1 of [19]. However, that proposition requires that the number of boundary components of the surface is minimal among all incompressible surfaces with the same boundary slope. In our case there is no guarantee that there is no higher genus surface with fewer boundary components of the same slope. Lemma 7.3 is probably false if there is no constraint about the number of components in $\partial F$.

Proof of Theorem 1.2. If $K_{\delta}$ is toroidal then clearly there is a toroidal incompressible surface in the exterior of $K$, so by Theorem 1.1 the pair $(K, \delta)$ must be one of those in the list.

To prove the other direction, we would like to show that if $(K, \delta)$ is in the list of Theorem 1.1 then the corresponding toroidal incompressible surface $F=F\left(\gamma_{1}, \gamma_{2}, \gamma_{3}\right)$ gives rise to an incompressible torus $\hat{F}$ in $K_{\delta}$. By Oertel [20], the exterior of $K\left(t_{1}, t_{2}, t_{3}\right)$ contains no closed essential surface. Therefore by Lemma 7.3 it suffices to show that $F$ has at most four boundary components.

Let $m_{i}$ be the number defined before the statement of Lemma 2.2, and let $n=\operatorname{lcm}\left(m_{1}, m_{2}, m_{3}\right)$. Then by Lemma $2.2(3)$ there is an orientable candidate surface $F=F\left(\gamma_{1}, \gamma_{2}, \gamma_{3}\right)$ with $|\partial F| \leq 2 n$. Since $F$ is toroidal, by choosing a component of $F$ if necessary we may assume that $F$ is connected, so it is of genus one. Therefore if $n \leq 2$ then by Lemma 7.3 the surface $\hat{F}$ is an incompressible torus in $K_{\delta}$ and we are done. By definition $m_{i}$ can be easily calculated from $\bar{u}$ and $E_{i}=\left\langle p_{i} / q_{i}, r_{i} / s_{i}\right\rangle$, which can be found in the proof of the corresponding lemma for that $(K, \delta)$. We leave it to the reader to check that $m_{i} \leq 2$ for all $i$ in all the cases listed in the theorem, except that $m_{3}=4$ in case (13). (For each individual knot, one may also use Dunfield's program [16] to calculate $n=\operatorname{lcm}\left(m_{1}, m_{2}, m_{3}\right)$, which is shown as "number of sheets" in the program.) Therefore Theorem 1.2 follows from Lemma 7.3, except in Case (13) of Theorem 1.1.

For Case (13), let $F^{\prime}=F^{\prime}\left(\gamma_{1}, \gamma_{2}, \gamma_{3}\right)$ be the surface in the exterior of $K$ constructed using the candidate system $\left(\gamma_{1}, \gamma_{2}, \gamma_{3}\right)$ given by Lemma 6.3 , such that $\left|\partial F^{\prime}\right|=n=4$. By the proof of Lemma 2.2(3) we have $F=F^{\prime}$, or 
its double cover if $F^{\prime}$ is nonorientable. In the first case we have $|\partial F|=4$ and the result follows from Lemma 7.3. Hence we assume that $\hat{F}$ is a double cover of $\hat{F}^{\prime}$, so $\hat{F}^{\prime}$ is a Klein bottle in $M=K_{\delta}$. On the other hand, from Theorem 1.1 we see that in this case $\delta=1$, hence $H_{1}\left(K_{\delta}, \mathbb{Z}_{2}\right)=0$, which is a contradiction because by duality a $\mathbb{Z}_{2}$-homology sphere cannot contain a Klein bottle.

\section{Acknowledgments}

I would like to thank Hatcher and Oertel for their algorithm in [4], which is crucial to the current work. Thanks also to the referee for some useful comments, and to Nathan Dunfield for his program [16], which has been used to verify that the slopes found in Theorem 1.1 are indeed toroidal boundary slopes. The author was partially supported by NSF grant no. DMS 0203394.

\section{References}

[1] G. Perelman, The entropy formula for the Ricci flow and its geometric applications, ArXiv:math.DG/0211159

[2] M. Brittenham and Y-Q. Wu, The classification of exceptional Dehn surgeries on 2-bridge knots, Comm. Anal. Geom. 9 (2001), 97-113.

[3] M. Eudave-Muñoz, Band sums of links which yield composite links. The cabling conjecture for strongly invertible knots, Trans. Amer. Math. Soc. 330 (1992), 463-501.

[4] A. Hatcher and U. Oertel, Boundary slopes for Montesinos knots, Topology 28 (1989), 453-480.

[5] Y-Q. Wu, Dehn surgery on arborescent knots, J. Diff. Geom. 42 (1996), 171-197.

[6] C. Gordon and J. Luecke, Non-integral toroidal Dehn surgeries, Commun. Anal. Geom. 12 (2004), 417-485.

[7] M. Eudave-Muñoz, Non-hyperbolic manifolds obtained by Dehn surgery on hyperbolic knots, Geometric topology (Athens, GA, 1993), AMS/IP Stud. Adv. Math. 2.1, Amer. Math. Soc. Providence, RI, 1997, pp. 35-61.

[8] C. Gordon, Boundary slopes of punctured tori in 3-manifolds, Trans. Amer. Math. Soc. 350 (1998), 1713-1790. 
[9] C. Gordon and J. Luecke, Dehn surgeries on knots creating essential tori, I, Commun. Anal. Geo. 3 (1995), 597-644.

[10] C. Gordon and J. Luecke, Toroidal and boundary-reducing Dehn fillings, Topology Appl. 93 (1999), 77-90.

[11] C. Gordon and Y-Q. Wu, Toroidal and annular Dehn fillings, Proc. London Math. Soc. 78 (1999), 662-700.

[12] S. Oh, Reducible and toroidal manifolds obtained by Dehn filling, Topology Appl. 75 (1997), 93-104.

[13] M. Teragaito, On hyperbolic knots realizing the maximal distance between toroidal surgeries, J. Knot Theory Ram. 15 (2006), 101-119.

[14] M. Teragaito, Distance between toroidal surgeries on hyperbolic knots in the 3-sphere, Trans. Amer. Math. Soc. 361 (2009), 3373-3374.

[15] Y-Q. Wu, Dehn fillings producing reducible manifolds and toroidal manifolds, Topology 37 (1998), 95-108.

[16] N. Dunfield, Program to compute the boundary slopes of a 2-bridge or Montesinos knot, available online at http://www.math.uiuc.edu/ nmd/montesinos/index.html.

[17] W. Jaco, Adding a 2-handle to 3-manifolds: An application to Property $R$, Proc. Amer. Math. Soc. 92 (1984), 288-292.

[18] A. Casson and C. Gordon, Reducing Heegaard splittings, Topology Appl. 27 (1987), 275-283.

[19] M. Culler, C. Gordon, J. Luecke and P. Shalen, Dehn surgery on knots, Ann. Math. 125 (1987), 237-300.

[20] U. Oertel, Closed incompressible surfaces in complements of star links, Pacific J. Math. 111 (1984), 209-230.

DePARTMENT OF MATHEMATiCs

UNIVERSITY OF IOWA

IOWA CiTy

IA 52242

USA

E-mail address: wu@math.uiowa.edu

RECEIVED JANUARY 16, 2011 
